\title{
Efficient End-fire Coupling of Surface Plasmons in a Metal Waveguide
}

May 7, 2015

Caitlin Fisher,$^{1^{*}}$ Lindsay C. Botten, ${ }^{2,3}$ Christopher G. Poulton, ${ }^{3}$ Ross C. McPhedran, ${ }^{1}$ and C. Martijn de Sterke $^{1}$

Centre for Ultrahigh bandwidth Devices for Optical Systems (CUDOS)

${ }^{1}$ School of Physics, University of Sydney, Sydney, Australia

${ }^{2}$ National Computational Infrastructure, Australian National University, Canberra, Australia

${ }^{3}$ School of Mathematical Sciences, University of Technology Sydney, Sydney, Australia

*Corresponding author: caitlin@physics.usyd.edu.au

\begin{abstract}
We present a semi-analytical study exploring the end-fire coupling of an incident beam into a surface plasmon mode propagating on a metal-dielectric interface. An energy-conserving projection method is used to solve for the resultant reflected and transmitted fields for a given incident beam, thereby determining the efficiency of the surface plasmon coupling. The coupling efficiency is found to be periodic with waveguide width due to the presence of a transversely propagating surface plasmon. Optimisation of the incident beam parameters, such as beam width, position and wavelength leads to numerically observed maximum efficiencies of approximately $80 \%$ when the beam width roughly matches the width of the surface plasmon.
\end{abstract}

OCID codes: (240.6680) Surface plasmons; (240.5420) Polaritons. 


\section{Introduction}

Surface plasmons (SPs) are localised field oscillations that propagate along the interface between a dielectric and a metal, attenuating into each medium. The propagating and attenuation characteristics of SPs are highly dependent on the properties of the interface and provide high field confinement, making them very useful for characterising surfaces, enhancing Raman spectroscopy and fluorescence sensing, photonic circuitry, signal processing and optical storage devices [1,2]. The characteristics and dispersion curves of SPs in single- and multiple-layered metal-dielectric structures have been reported extensively [3-5]. The two most commonly used coupling methods are prism coupling and grating coupling. Prism coupling involves coupling evanescent light from a high-refractive index prism into the SP mode via either the Otto [6] or the Kretschmann-Raether [7] configuration, so that the parallel wavevector component of light matches that of the SP mode. On the other hand, grating coupling requires a grating to satisfy the coupling condition that the parallel wavevector of the incident light and the SP only differ by a multiple of the reciprocal lattice vector [8]. A disadvantage of these two methods is that the frequency of the incident light is limited by wave-vector matching constraints. The prism coupling method in particular is also bulky and does not lend itself well to on-chip applications [9].

A more broadband coupling technique is the end-fire coupling mechanism [10], where the incident light is aligned parallel to the metal-dielectric interface, and where the coupling results from having sufficient overlap of the incident field with the SP field [2]. Stegeman et al. theoretically demonstrated that end-fire coupling can couple an incident beam with wavelength $\lambda=0.5 \mu \mathrm{m}$ into a SP along an air and losslesssilver interface with efficiencies of up to approximately 90\% [10]. End-fire coupling was then successfully used in the laboratory by Charbonneau et al. in 2000 to couple $\lambda=1.55 \mu \mathrm{m}$ wavelength light onto a $3.55 \mu \mathrm{m} \mathrm{Au} \mathrm{strip} \mathrm{on} \mathrm{SiO}_{2}$ [11]. Since then, end-fire coupling has been shown to be an effective way of coupling SPs into more complex geometries, such as into waveguide-like nanocavities [12], in and out of dielectric and plasmonic waveguides [13-18], between nanowires and nanofibres $[19,20]$, and photonic crystal fibres [21]. SP modes inside the finite nanocavities were studied theoretically by Al-Bader et al. [22] and then explored both theoretically and experimentally by Kurokawa et al. [12], though neither modelled the end-fire coupling step. Charbonneau et al., in particular, have experimentally demonstrated successful end-fire coupling in a wide variety of geometries including bent waveguides, Y-junctions, Mach-Zehnder interferometers, and four-port couplers all for incident 
waveguide of $1.5 \mu \mathrm{m}[23,24]$. Hu et al. have recently shown end-fire coupling of SPs to improve the performance of a plasmonic Mach-Zehnder interferometer [25].

While there have been many theoretical studies on the fundamental characteristics and properties of SPs, and also the coupling of SP modes into other SP modes, few of them address the end-fire coupling mechanism of a laser into a SP mode. The original theoretical study on end-fire coupling, conducted by Stegeman et al., was based on a semi-analytic collocation method which modelled the incident, reflected and transmitted fields as a superposition of discretised waveguide modes $[10,26]$. They chose their geometry to consist of lossless media and used $P=60-80$ modes to approximate the electromagnetic fields. The incident field was approximated by a top hat-function, and the reflection and transmission coefficients were solved by using a collocation method which ensured the continuity of the transverse fields at a fixed number of data points $P$ along the coupling interface.

This paper aims to revisit the fundamental end-fire coupling mechanism in order to deepen our understanding of what determines the SP coupling efficiency and how it can be maximised. By using a sufficient number of modes (see Eqs (52) and (53) in Section 2.4), we numerically observe a phenomenon unseen by Stegeman et al. [10]: a transversely propagating SP which occurs despite not specifically being built into the model. The presence of the transverse-SP greatly affects the coupling efficiency of the forward-propagating SP, which we will demonstrate in this work.

Instead of using the collocation method, our calculations are made using both a least-squares method and a projection method, which enable the use of different numbers of modes in the incident and transmitted medium. While the least-squares method, like the collocation method, matches fields at chosen points, the projection method matches fields across a continuous range of points. The least-squares method is not discussed in this paper as the method is a standard one and commonly used, and, more importantly, yields the same results as the projection method, which is discussed in detail in this paper.

In Section 2, we model the end-fire coupling mechanism using the geometry proposed by Stegeman et al. [10]. This geometry is described in detail in Section 2.1, and consists of a perfectly grounded two-dimensional waveguide split into two regions; Region I is filled with a homogeneous dielectric, and Region II is filled with a layer of dielectric on top of a layer of lossless metal. In Sections 2.2 and 2.3, we outline the modes in Regions I and II, and in Section 2.4 discuss the derivation and properties of the projection method used to calculate the reflected and transmitted fields of the configuration. In Section 3, we determine the SP coupling efficiency 
dependence on waveguide width, the dielectric permittivities, the ratios of metaldielectric widths in Region II and various parameters of the incident beam. We summarise our conclusions from this work in Section 4.

\section{Method}

\subsection{Coupling Geometry}

The end-fire coupling configuration, as seen in Fig. 1, consists of a beam of wavelength $\lambda$ which is incident onto a metal-dielectric interface. The configuration is bounded by a perfectly grounded metal waveguide. The role of the waveguide is to provide discretised modes, allowing the electric and magnetic fields of the configuration to be defined with analytical ease. The wavenumber of the incident light in vacuum is $k_{0}=2 \pi / \lambda$, corresponding to an angular frequency of $\omega=k_{0} c$, where $c$ is the speed of light in vacuum. The two-dimensional waveguide extends infinitely in the $y$-direction and has walls at $x= \pm l$. This two-dimensional configuration can easily be generalised to the three-dimensional case if the width in the y-direction is much larger than the diffraction of the SP. This has been assumed in several other theoretical papers $[12,15]$. The incident beam propagates from Region $\mathrm{I}(z<0)$, which is filled with a semi-infinite dielectric medium between $-l<x<l$ with permittivity $\varepsilon_{\mathrm{dI}}$. Region II $(z>0)$ contains two layers of semi-infinite media: a dielectric between $\Delta l<x<l$ with permittivity $\varepsilon_{\mathrm{dII}}$ and a lossless metal between $-l<x<\Delta l$ with permittivity $\varepsilon_{\mathrm{m}}$. The widths of the Region II dielectric and the metal are

$$
\begin{gathered}
l_{\mathrm{d}}=l-\Delta l, \\
l_{\mathrm{m}}=l+\Delta l,
\end{gathered}
$$

respectively. The metal permittivity is approximated by the lossless Drude model, $\varepsilon_{\mathrm{m}}(\lambda)=1-\left(\lambda / \lambda_{\mathrm{p}}\right)^{2}$, where $\lambda_{\mathrm{p}}$ is the plasmon wavelength characteristic of the metal. The SP field is localised on the surface of the metal-dielectric interface in Region II $(x=0, z>0)$ propagating in the $z$-direction and decaying in both Region II media. In order to be able to couple into the SP mode, the incident light must be transverse magnetic, where the magnetic field is orthogonal to the plane of the incident field. Thus the only field components are the magnetic field in the $y$-direction $\left(H_{y}\right)$, and the electric field components in the $x$ - and $z$-direction $\left(E_{x}\right.$ and $E_{z}$ respectively). We must have $E_{\mathrm{z}}=0$ at the grounded walls, $x= \pm l$. 
The one-dimensional scalar wave equation, derived from the Maxwell curl equations, is

$$
\frac{\partial}{\partial x} \frac{1}{\varepsilon}\left(\frac{\partial H_{y}}{\partial x}\right)+k_{0}^{2} H_{y}=\frac{\beta^{2}}{\varepsilon} H_{y},
$$

for a magnetic field in which $\mathbf{H}=H_{y} \exp (i \beta z) \hat{y}$. Here $H_{y}$ is the scalar amplitude of the magnetic field, $\beta$ is the propagation constant, $\varepsilon$ is the relative (positiondependent) permittivity of the medium, the relative permeability is assumed to be $\mu=1$, and an $\exp (-i \omega t)$ time dependence was taken. The incident and reflected fields are expanded in terms of the Region I modes, and likewise the transmitted field is expanded in terms of Region II modes. These modes are discussed in Sections 2.2 and 2.3 .

\subsection{Region I Modes}

In this section, we derive the Region I modes required for expanding the incident and reflected magnetic and electric fields. The $p^{t h}$ mode for the forward-propagating magnetic field in Region I $(z<0)$ is expressed as

$$
\hat{H}_{y p}^{\mathrm{I}+}=\frac{1}{\sqrt{\chi_{p}^{\mathrm{I}}}} \mathcal{H}_{p}^{\mathrm{I}}(x) \exp \left(i \beta_{p}^{\mathrm{I}} z\right)
$$

where

$$
\mathcal{H}_{p}^{\mathrm{I}}(x)=\left[\frac{\varepsilon_{\mathrm{dI}}}{\left(1+\delta_{p 0}\right) l}\right]^{\frac{1}{2}} \cos \left(\gamma_{p}(x-l)\right),
$$

where $\delta$ is the Kronecker-delta function, and $\chi$ has been introduced for notational convenience,

$$
\chi_{p}^{\mathrm{I}}=\frac{\beta_{p}^{\mathrm{I}}}{k_{0}} .
$$

In Eq. (4) and for the rest of this work, we define $\hat{H}=Z_{0} H$, where $Z_{0}$ is the impedance of free space. The $\beta^{\mathrm{I}}$ are the propagation constants in the $z$-direction, and $\gamma$ are the complex spatial frequencies in the $x$-direction. The $x$ - and $z$-components of the electric field can be derived from $\hat{H}_{y p}^{\mathrm{I}+}$ by one of the Maxwell curl equation, and the $p^{\text {th }}$ mode of each can be expressed as

$$
E_{x p}^{\mathrm{I}+}=\sqrt{\chi_{p}^{\mathrm{I}}} \mathcal{E}_{p}^{\mathrm{I}}(x) \exp \left(i \beta_{p}^{\mathrm{I}} z\right)
$$


where

$$
\mathcal{E}_{p}^{\mathrm{I}}(x)=\frac{\mathcal{H}_{p}^{\mathrm{I}}(x)}{\varepsilon_{\mathrm{dI}}},
$$

and

$$
E_{z p}^{I+}=-\frac{\gamma_{p}}{\left[\left(1+\delta_{p 0}\right) l \chi_{p}^{\mathrm{I}} \varepsilon_{\mathrm{dI}}\right]^{\frac{1}{2}}} \sin \left(\gamma_{p}(x-l)\right) \exp \left(i \beta_{p}^{\mathrm{I}} z\right)
$$

The boundary condition for a perfect conductor, $E_{z p}^{\mathrm{I}+}(x=l)=0$, is satisfied by the form of Eq. (9). In order for $E_{z p}^{\mathrm{I}+}(x=-l)=0$ to be satisfied also, then

$$
\gamma_{p}=\frac{p \pi}{2 l}, \text { for } p=0,1 \ldots
$$

The wave equation (3) relates $\beta^{\mathrm{I}}$ to $\gamma$ by

$$
\beta_{p}^{\mathrm{I}}=\left(\varepsilon_{\mathrm{dI}} k_{0}^{2}-\gamma_{p}^{2}\right)^{\frac{1}{2}}
$$

The $\beta^{\mathrm{I}}$ are real for propagating modes and imaginary for evanescent modes. The $\mathcal{H}_{p}^{\mathrm{I}}(x)$ and $\mathcal{E}_{\mathrm{p}}^{\mathrm{I}}(x)$ functions have been normalised so that

$$
\int_{-l}^{l} \mathcal{H}_{p}^{\mathrm{I}} \mathcal{E}_{p^{\prime}}^{\mathrm{I}} d x=\delta_{p p^{\prime}}
$$

The $p^{\text {th }}$ mode of the backward propagating magnetic field for Region I is

$$
\hat{H}_{y p}^{\mathrm{I}-}=\frac{1}{\sqrt{\chi_{p}^{\mathrm{I}}}} \mathcal{H}_{p}^{\mathrm{I}}(x) \exp \left(-i \beta_{p}^{\mathrm{I}} z\right)
$$

which differs from the forward propagating modes by a minus sign in the exponential factor. Similarly, the backward propagating modes of the electric field components are

$$
E_{x p}^{\mathrm{I}-}=-\sqrt{\chi_{p}^{\mathrm{I}}} \mathcal{E}_{p}^{\mathrm{I}}(x) \exp \left(-i \beta_{p}^{\mathrm{I}} z\right)
$$

and

$$
E_{z p}^{\mathrm{I}-}=-\frac{\gamma_{p}}{\left[\left(1+\delta_{p 0}\right) l \chi_{p}^{\mathrm{I}} \varepsilon_{\mathrm{dI}}\right]^{\frac{1}{2}}} \sin \left(\gamma_{p}(x-l)\right) \exp \left(-i \beta_{p}^{\mathrm{I}} z\right)
$$




\section{$2.3 \quad$ Region II Modes}

In this section, we derive the Region II modes required to expand the transmitted magnetic and electric fields. The $q^{\text {th }}$ mode of the forward-propagating magnetic field in Region II $(z>0)$ can be expressed as

$$
\hat{H}_{y q}^{\mathrm{II}+}=\frac{1}{\sqrt{\chi_{q}^{\mathrm{II}}}} \mathcal{H}_{q}^{\mathrm{II}}(x) \exp \left(i \beta_{q}^{\mathrm{II}} z\right),
$$

where $\chi^{\mathrm{II}}$ is the Region II counterpart of $\chi^{\mathrm{I}}$ in Eq. (6). The $\beta^{\mathrm{II}}$ are the propagation constants of Region II in the positive $z$-direction. The $\mathcal{H}_{q}^{\mathrm{II}}(x)$ is of the form

$$
\mathcal{H}_{q}^{\mathrm{II}}(x)= \begin{cases}N_{q}^{\mathrm{II}} \frac{\cosh \left(\widetilde{\gamma}_{\mathrm{d} q}(x-l)\right)}{\cosh \left(\widetilde{\gamma}_{\mathrm{d} q} l_{\mathrm{d}}\right)}, & \Delta l<x<l, \\ N_{q}^{\mathrm{II}} \frac{\cosh \left(\widetilde{\gamma}_{\mathrm{m} q}(x+l)\right)}{\cosh \left(\widetilde{\gamma}_{\mathrm{m} q} l_{\mathrm{m}}\right)}, & -l<x<\Delta l,\end{cases}
$$

where the $N_{q}^{\mathrm{II}}$ are normalisation coefficients and $\widetilde{\gamma}_{\mathrm{d}}$ and $\widetilde{\gamma}_{\mathrm{m}}$ are the complex spatial frequencies in the $\pm x$-directions, that is, into the dielectric and metal respectively. The electric field components are determined from the Maxwell equations, and the resulting $q^{\text {th }}$ mode of the $x$ - and $z$-components are

$$
E_{x q}^{\mathrm{II}+}=\sqrt{\chi_{q}^{\mathrm{II}}} \mathcal{E}_{q}^{I I}(x) \exp \left(i \beta_{q}^{\mathrm{II}} z\right)
$$

where

$$
\mathcal{E}_{q}^{\mathrm{II}}= \begin{cases}\frac{\mathcal{H}_{q}^{\mathrm{II}}}{\varepsilon_{\mathrm{dII}}}, & \Delta l<x<l, \\ \frac{\mathcal{H}_{q}^{\mathrm{II}}}{\varepsilon_{\mathrm{m}}}, & -l<x<\Delta l,\end{cases}
$$

and

$$
E_{z q}^{\mathrm{II}+}= \begin{cases}-\frac{\widetilde{\gamma}_{\mathrm{d} q}}{\varepsilon_{\mathrm{dII}}} \frac{N_{q}^{I I}}{\sqrt{\chi_{q}^{\mathrm{II}}}} \frac{\sinh \left(\widetilde{\gamma}_{\mathrm{d} q}\left(x-l_{\mathrm{d}}\right)\right)}{\cosh \left(\widetilde{\gamma}_{\mathrm{d} q} l_{\mathrm{d}}\right)}, \quad \Delta l<x<l, \\ -\frac{\widetilde{\gamma}_{\mathrm{d} q}}{\varepsilon_{\mathrm{m}}} \frac{N_{q}^{I I}}{\sqrt{\chi_{q}^{\mathrm{II}}}} \frac{\sinh \left(\widetilde{\gamma}_{\mathrm{m} q}\left(x+l_{\mathrm{m}}\right)\right)}{\cosh \left(\widetilde{\gamma}_{\mathrm{m} q} l_{\mathrm{m}}\right)}, & -l<x<\Delta l .\end{cases}
$$


The form of $\mathcal{H}_{q}^{\mathrm{II}}(x)$ in Eq. (17) which carries through into the form of Eq. (20), satisfies the condition $E_{z q}^{\mathrm{II}+}=0$ at $x= \pm l$. The normalisation factor $N_{q}^{\mathrm{II}}$ is defined so that the normalisation condition

$$
\int_{-l}^{l} \mathcal{H}_{q}^{\mathrm{II}}(x) \mathcal{E}_{q^{\prime}}^{\mathrm{II}}(x) d x=\delta_{q q^{\prime}},
$$

is satisfied, which requires

$$
\begin{array}{r}
N_{q}^{\mathrm{II}}=\left(\frac{\tanh \left(\widetilde{\gamma}_{\mathrm{d} q} l_{\mathrm{d}}\right)+\widetilde{\gamma}_{\mathrm{d} q} l_{\mathrm{d}} \operatorname{sech}^{2}\left(\widetilde{\gamma}_{\mathrm{d} q} l_{\mathrm{d}}\right)}{2 \widetilde{\gamma}_{\mathrm{d} q} \varepsilon_{\mathrm{dII}}}\right. \\
\left.+\frac{\tanh \left(\widetilde{\gamma}_{\mathrm{m} q} l_{\mathrm{m}}\right)+\widetilde{\gamma}_{\mathrm{m} q} l_{\mathrm{m}} \operatorname{sech}^{2}\left(\widetilde{\gamma}_{\mathrm{m} q} l_{\mathrm{m}}\right)}{2 \widetilde{\gamma}_{\mathrm{m} q} \varepsilon_{\mathrm{m}}}\right)^{-\frac{1}{2}} .
\end{array}
$$

The $\beta^{\mathrm{II}}$ values are determined from the condition that the magnetic and transverse electric fields are continuous over the metal-dielectric interface at $x=0$, or

$$
\begin{aligned}
\hat{H}_{y}^{\mathrm{II}+}\left(x=\Delta l^{-}\right) & =\hat{H}_{y}^{\mathrm{II}+}\left(x=\Delta l^{+}\right), \\
E_{x}^{\mathrm{II}+}\left(x=\Delta l^{-}\right) & =E_{x}^{\mathrm{II}+}\left(x=\Delta l^{+}\right) .
\end{aligned}
$$

After cancelling constants and similar $z$-dependent factors, this pair of simultaneous equations lead to the transcendental equation

$$
\frac{\widetilde{\gamma}_{\mathrm{d} q}}{\varepsilon_{\mathrm{dII}}} \tanh \left(\widetilde{\gamma}_{\mathrm{d} q} l_{\mathrm{d}}\right)+\frac{\widetilde{\gamma}_{\mathrm{m} q}}{\varepsilon_{\mathrm{m}}} \tanh \left(\widetilde{\gamma}_{\mathrm{m} q} l_{\mathrm{m}}\right)=0
$$

which can be solved to find $\beta^{\mathrm{II}}$. Again, the $\beta^{\mathrm{II}}$ relate to the $\widetilde{\gamma}_{\mathrm{d}}$ and $\widetilde{\gamma}_{\mathrm{m}}$ by the wave equation (3), giving

$$
\begin{aligned}
& \widetilde{\gamma}_{\mathrm{d} q}=\left[\left(\beta_{q}^{\mathrm{II}}\right)^{2}-\varepsilon_{\mathrm{dII}} k_{0}^{2}\right]^{\frac{1}{2}}, \\
& \widetilde{\gamma}_{\mathrm{m} q}=\left[\left(\beta_{q}^{\mathrm{II}}\right)^{2}-\varepsilon_{\mathrm{m}} k_{0}^{2}\right]^{\frac{1}{2}},
\end{aligned}
$$

respectively. There are four types of modes which are outlined in Table 1. Modes for which $\beta^{\mathrm{II}}$ is real are propagating modes, and modes for which $\beta^{\mathrm{II}}$ is imaginary 
or complex are evanescent modes. Modes for which $\widetilde{\gamma}_{\mathrm{d}}$ or $\widetilde{\gamma}_{\mathrm{m}}$ are real decay into their respective media, and modes for which $\widetilde{\gamma}_{\mathrm{d}}$ or $\widetilde{\gamma}_{\mathrm{m}}$ are imaginary or complex are oscillatory into their respective media. Case I is the single SP mode. There are a finite number of case II and III modes, and there are infinitely many case IV modes. It is worth noting that in case IV the $\left(\beta^{\mathrm{II}}\right)^{2}$ are either real and negative or come in complex conjugate pairs (with negative real parts), and thus that $\beta^{\mathrm{II}}$ can be either imaginary or come in complex pairs with positive imaginary parts and opposite signs of the real parts. These complex pairs occur in this lossless configuration as the $x$ dependence of $\varepsilon$ on the right-hand side in Eq. (3) causes the scalar differential wave equation to be non-Hermitian [27, 28].

We note that as $l_{\mathrm{d}, \mathrm{m}} \rightarrow \infty$, Eq. (25) reduces to the standard transcendental equation for a SP

$$
\frac{\widetilde{\gamma}_{\mathrm{d}}}{\varepsilon_{\mathrm{d}}}+\frac{\widetilde{\gamma}_{\mathrm{m}}}{\varepsilon_{\mathrm{m}}}=0
$$

which only has solutions if $\varepsilon_{\mathrm{m}}<-\varepsilon_{\mathrm{d}}[29]$.

\subsection{Projection method}

The incident magnetic field can be written as a summation of the modes of Region I, i.e

$$
\hat{H}_{y}^{\mathrm{I}+}=\sum_{p=0}^{P} \alpha_{p} \hat{H}_{y p}^{\mathrm{I}+}
$$

where the $\alpha_{p}$ are the incident amplitudes which characterise the incident beam. Similar expressions to Eq. (29) can be defined for the incident electric field components, $E_{x}^{\mathrm{I}+}$ and $E_{z}^{\mathrm{I}+}$. For the purpose of analytical calculation, the Region I modes in the series of Eq. (29) must be truncated to a finite number $P$.

In this work, the incident beam amplitudes, $\alpha_{p}$, are chosen so that $E_{x}^{\mathrm{I}+}$ is a Gaussian at $z=0$, with peak at $x=l_{\mathrm{G}}$ and full width of the field $w$ at $1 / e$ of the peak, so that the full width half maximum of the intensity is $w \sqrt{(\ln 2) / 2}$. The expression for the Gaussian electric field is therefore

$$
E_{x}^{\mathrm{I}+}=\exp \left[-\left(\frac{2\left(x-l_{\mathrm{G}}\right)}{w}\right)^{2}\right] .
$$


This requires the incident amplitudes to be

$$
\alpha_{p}= \begin{cases}\frac{\omega}{4}\left(\frac{\pi\left(1+\delta_{p 0}\right)}{l \chi_{p}^{\mathrm{I}} \varepsilon_{d l}}\right)^{\frac{1}{2}}, & p=0, \\ \frac{\omega}{2}\left(\frac{\pi\left(1+\delta_{p 0}\right)}{l \chi_{p}^{\mathrm{I}} \varepsilon_{d l}}\right)^{\frac{1}{2}} \exp \left(-\left(\frac{\gamma_{p} \omega}{4}\right)^{2}\right) \cos \left(\gamma_{p}\left(l_{G}-l\right)\right), & p=1,2 \ldots\end{cases}
$$

where we have taken the incident beam to be well contained within the waveguide (i.e. $w \ll l$ ). Likewise, the reflected magnetic field can be written as a summation of backward-propagating Region I modes, and in our notation is given by

$$
\hat{H}_{y}^{\mathrm{I}-}=\sum_{p=0}^{P} r_{p} \hat{H}_{y p}^{\mathrm{I}-},
$$

where the $r$ are the unknown reflection amplitudes of each mode. Similar expressions to Eq. (32) can be defined for the reflected electric field components, $E_{x}^{\mathrm{I}-}$ and $E_{z}^{\mathrm{I}-}$. Finally, the transmitted magnetic field can be written as a summation of the set of forward-propagating Region II modes, given in our notation as

$$
\hat{H}_{y}^{\mathrm{II}+}=\sum_{q=1}^{Q} t_{q} \hat{H}_{y q}^{\mathrm{II}+}
$$

where $t$ are the unknown transmission amplitudes of each mode, and the series is truncated at $Q$ modes. Similar expressions can be defined for the transmitted electric field components, $E_{x}^{\mathrm{II}+}$ and $E_{z}^{\mathrm{II}+}$. At full rank, that is, when $P, Q \rightarrow \infty$, the continuity of $H_{y}$ and $E_{x}$ over $z=0$ can be expressed as

$$
\begin{gathered}
\hat{H}_{y}^{\mathrm{I}+}+\hat{H}_{y}^{\mathrm{I}-}=\hat{H}_{y}^{\mathrm{II}+}, \\
E_{x}^{\mathrm{I}+}+E_{x}^{\mathrm{I}-}=E_{x}^{\mathrm{II}+} .
\end{gathered}
$$

The unknown reflection coefficients, $r$, and transmission coefficients, $t$, are solved such that these conditions are satisfied for a truncated set of modes in the leastsquares sense. Here, we describe a projection method to solve this scattering problem. The key to the method lies in having two sets of orthogonal basis functions, corresponding to the modes in Regions I and II. 
Expanding the Eqs (34) in terms of Eqs (4), (13) and (16), and likewise expanding (35) in terms of Eqs (7), (14) and (20) at $z=0$ gives

$$
\begin{gathered}
\sum_{p=0}^{P}\left(\chi_{p}^{\mathrm{I}}\right)^{-\frac{1}{2}}\left(\alpha_{p}+r_{p}\right) \mathcal{H}_{p}^{\mathrm{I}}=\sum_{q=1}^{Q}\left(\chi_{q}^{\mathrm{II}}\right)^{-\frac{1}{2}} t_{q} \mathcal{H}_{q}^{\mathrm{II}}, \\
\sum_{p=0}^{P}\left(\chi_{p}^{\mathrm{I}}\right)^{\frac{1}{2}}\left(\alpha_{p}-r_{p}\right) \mathcal{E}_{p}^{\mathrm{I}}=\sum_{q=1}^{Q}\left(\chi_{q}^{\mathrm{II}}\right)^{\frac{1}{2}} t_{q} \mathcal{E}_{q}^{\mathrm{II}},
\end{gathered}
$$

from which we can obtain expressions for the unknown $r_{p}$ and $t_{q}$ by projection. We obtain two different but equivalent formations by projecting Eqs (36), (37) onto $\mathcal{E}^{\mathrm{I}}$ and $\mathcal{H}^{\mathrm{II}}$, and $\mathcal{E}^{\mathrm{II}}$ onto $\mathcal{H}^{\mathrm{I}}$, respectively.

Proceeding with the first of these formulations, we multiply Eq. (36) by $\mathcal{E}_{p^{\prime}}^{\mathrm{I}}$ and integrate from $x=-l$ to $x=l$,

$$
\sum_{p=0}^{P}\left(\chi_{p}^{\mathrm{I}}\right)^{-\frac{1}{2}}\left(\alpha_{p}+r_{p}\right) \int_{-l}^{l} \mathcal{E}_{p}^{\mathrm{I}} \mathcal{H}_{p^{\prime}}^{\mathrm{I}} d x=\sum_{q=1}^{Q}\left(\chi_{q}^{\mathrm{II}}\right)^{-\frac{1}{2}} t_{q} \int_{-l}^{l} \mathcal{E}_{p^{\prime}}^{\mathrm{I}} \mathcal{H}_{q}^{\mathrm{II}} d x
$$

By using orthogonality condition in Eq. (12) and with some rearranging, Eq. (38) can be simplified to

$$
\alpha_{p^{\prime}}+r_{p^{\prime}}=\sum_{q=1}^{Q}\left(\chi_{p^{\prime}}^{\mathrm{I}}\right)^{\frac{1}{2}} J_{p^{\prime} q}\left(\chi_{q}^{\mathrm{II}}\right)^{-\frac{1}{2}} t_{q}
$$

where the matrix $J$ has been defined so that the $(p q)^{t h}$ element is

$$
J_{p q}=\int_{-l}^{l} \mathcal{E}_{p}^{\mathrm{I}} \mathcal{H}_{q}^{\mathrm{II}} d x
$$

To express Eq. (39) in matrix notation, let $\boldsymbol{\alpha}, \boldsymbol{r}$ and $\boldsymbol{t}$ be column vectors of the modal amplitudes and let $\chi^{\mathrm{I}}$ and $\chi^{\mathrm{II}}$ be square matrices with their respective $\chi$ values on their diagonals. The matrix formulation of Eq. (39) then becomes

$$
(\boldsymbol{\alpha}+\boldsymbol{r})=\left(\chi^{\mathrm{I}}\right)^{\frac{1}{2}} \boldsymbol{J}\left(\chi^{\mathrm{II}}\right)^{-\frac{1}{2}} \boldsymbol{t}
$$


Similarly, an expression for $\boldsymbol{t}$ is derived by projecting Eq. (37) onto $\mathcal{H}_{q^{\prime}}^{\mathrm{II}}$ and simplifying using the orthogonality relation (21), and the expression becomes

$$
\left(\chi^{\mathrm{II}}\right)^{-\frac{1}{2}} \boldsymbol{J}^{T}\left(\chi^{\mathrm{I}}\right)^{\frac{1}{2}}(\boldsymbol{\alpha}-\boldsymbol{r})=\boldsymbol{t}
$$

By simultaneously solving Eqs (41) and (42) the expressions for $\boldsymbol{r}$ and $\boldsymbol{t}$ are found to be

$$
\begin{aligned}
\boldsymbol{r} & =\left(\boldsymbol{I}+\boldsymbol{A} \boldsymbol{A}^{T}\right)^{-1}\left(\boldsymbol{A} \boldsymbol{A}^{T}-\boldsymbol{I}\right) \boldsymbol{\alpha} \equiv \boldsymbol{R} \boldsymbol{\alpha} \\
\boldsymbol{t} & =2 \boldsymbol{A}^{T}\left(\boldsymbol{I}+\boldsymbol{A} \boldsymbol{A}^{T}\right)^{-1} \boldsymbol{\alpha} \equiv \boldsymbol{T} \boldsymbol{\alpha}
\end{aligned}
$$

where

$$
\boldsymbol{A}=\left(\chi^{\mathrm{I}}\right)^{\frac{1}{2}} \boldsymbol{J}\left(\chi^{\mathrm{II}}\right)^{-\frac{1}{2}}
$$

It may be noted that the $\boldsymbol{r}$ and $\boldsymbol{t}$ equations are reminiscent of the standard Fresnel coefficients.

An alternative set of expressions for $\boldsymbol{r}$ and $\boldsymbol{t}$ can be derived by using the opposite set of projections. Taking the projections of Eq. (36) onto $\mathcal{E}_{q^{\prime}}^{\mathrm{II}}$ and of Eq. (37) onto $\mathcal{H}_{p^{\prime}}^{\mathrm{I}}$, we find

$$
\begin{gathered}
(\boldsymbol{\alpha}-\boldsymbol{r})=\left(\boldsymbol{\chi}^{\mathrm{I}}\right)^{-\frac{1}{2}} \boldsymbol{K}\left(\boldsymbol{\chi}^{\mathrm{II}}\right)^{\frac{1}{2}} \boldsymbol{t}, \\
\left(\chi^{\mathrm{II}}\right)^{\frac{1}{2}} \boldsymbol{K}^{T}\left(\chi^{\mathrm{I}}\right)^{-\frac{1}{2}}(\boldsymbol{\alpha}+\boldsymbol{r})=\boldsymbol{t} .
\end{gathered}
$$

Here $\boldsymbol{K}^{T}$ is the transpose of $\boldsymbol{K}$, where the $\boldsymbol{K}$ matrix is defined to have a $(p q)^{t h}$ term

$$
K_{p q}=\int_{-l}^{l} \mathcal{E}_{q}^{\mathrm{II}} \mathcal{H}_{p}^{\mathrm{I}} d x
$$

Using the same procedure is before we then find that

$$
\begin{aligned}
\boldsymbol{r} & =\left(\boldsymbol{I}+\boldsymbol{B}^{T} \boldsymbol{B}\right)^{-1}\left(\boldsymbol{I}-\boldsymbol{B}^{T} \boldsymbol{B}\right) \boldsymbol{\alpha} \equiv \boldsymbol{R} \boldsymbol{\alpha} \\
\boldsymbol{t} & =2 \boldsymbol{B}\left(\boldsymbol{I}+\boldsymbol{B}^{T} \boldsymbol{B}\right)^{-1} \boldsymbol{\alpha} \equiv \boldsymbol{T} \boldsymbol{\alpha}
\end{aligned}
$$

where

$$
\boldsymbol{B}=\left(\chi^{\mathrm{II}}\right)^{\frac{1}{2}} \boldsymbol{K}^{T}\left(\chi^{\mathrm{I}}\right)^{-\frac{1}{2}},
$$

which are equivalent to Eqs (43)-(45).

In order to reconcile the two sets of formulations, (43) and (44) with (49) and (50) we must recognise the completeness relations 


$$
\boldsymbol{J}^{T} \boldsymbol{K}=\boldsymbol{I},
$$

and

$$
\boldsymbol{J} \boldsymbol{K}^{T}=\boldsymbol{I}
$$

which apply at full rank and which are derived in the Appendix. These relations show that $\boldsymbol{B}=\boldsymbol{A}^{-1}$ at full rank, which can be used to demonstrate the equivalence of the two formulations (see Appendix).

The completeness relations (52) and (53) provide a useful measure of how well represented the modes in one region are when expanded in terms of the truncated set of modes in the other region [30]. In particular, the closer $\boldsymbol{J}^{T} \boldsymbol{K}$ in (52) is to the identity matrix, the more accurate the expansion of each Region I modes in terms of the modes in Region II replicates the property that the inner products of the mode with: (a) itself produces unity (diagonal elements), and (b) with all other modes produces zero (off-diagonal elements). Similarly, the completeness relation (53) serves as a measure of how well represented each Region II mode is when expanded in terms of the truncated set of Region I modes.

The energy conservation equation along the interface $z=0$ is derived from integrating the time-averaged Poynting vector between $-l<x<l$, and is expressed as

$$
S_{z}=2 \operatorname{Re}\left\{\int_{-l}^{l} E_{x} H_{y}^{*} d x\right\},
$$

on either side of the interface $(z=0)$. The calculation of the energy flux for each region is given in the Appendix. Equating the energy flux of each region on either side of the $(z=0)$ interface gives an expression for the conservation of energy;

$$
\sum_{p \in \Omega_{\mathrm{P}}^{\mathrm{I}}}\left|\alpha_{p}\right|^{2}=\sum_{p \in \Omega_{\mathrm{p}}^{\mathrm{I}}}\left|r_{p}\right|^{2}-2 \operatorname{Im} \sum_{p \in \Omega_{\mathrm{e}}^{\mathrm{I}}} \alpha_{p} r_{p}^{*}+\sum_{q \in \Omega_{\mathrm{P}}^{\mathrm{II}}}\left|t_{q}\right|^{2} .
$$

The energy flux conservation equation notably includes a contribution from the propagating modes from both Regions I $\left(\Omega_{\mathrm{p}}^{\mathrm{I}}\right)$ and II $\left(\Omega_{\mathrm{p}}^{\mathrm{II}}\right)$, and also from the evanescent modes $\left(\Omega_{\mathrm{e}}^{\mathrm{I}}\right)$ of Region I. The evanescent energy contribution includes the possibility that the incident beam is a near-field source with significant contributions from its 
evanescent modes. Evanescent mode amplitudes are significant in narrow incident beams, allowing them to contribute to the energy flow. However, if the beam is narrow far away from the interface, then the evanescent fields decay before reaching the interface, causing the evanescent energy contribution to be negligible.

In this work with lossless media, the energy flux relation in Eq. (55) is analytically satisfied regardless of mode truncation. In contrast, for a structure with loss, the energy flux relation is satisfied analytically, but only with a complete (i.e. infinite) set of modes. The extent of the defect in the flux relation would serve as a measure of the truncation error [31].

The SP coupling efficiency $\eta$ is determined by taking the transmission amplitude that corresponds to the SP and normalising it by the total incident energy, and is expressed as

$$
\eta=\frac{\left|t_{S P}\right|^{2}}{\left(\sum_{p \in \Omega_{\mathrm{p}}^{\mathrm{I}}}\left|\alpha_{p}\right|^{2}-2 \operatorname{Im} \sum_{p \in \Omega_{\mathrm{e}}^{\mathrm{I}}} \alpha_{p} r_{p}^{*}\right)} .
$$

\subsubsection{Completeness tests}

The degree to which mode truncation leads to deviations from the completeness equations (52) and (53) are illustrated in Figs 2a and 2b. These figures show the logarithm base 10 of the absolute values of each of the elements, respectively, of the matrices $\boldsymbol{J}^{T} \boldsymbol{K}-\boldsymbol{I}$, which is a $Q \times Q$ matrix, and $\boldsymbol{J} \boldsymbol{K}^{T}-\boldsymbol{I}$, which is $P \times P$, for a geometry with parameters given in the caption. The figures show that the first $70 \%$ of entries on the diagonal are equal to unity to within $10^{-3}$ and that the offdiagonal cross-elements of these entries are equal to zero to within $10^{-5}$. Evidently the low-order modes in one region can be represented, predominantly, by low-order modes in the other region, so that the truncation does not matter for these modes. Note that the abrupt change which occurs at $q=144$ in Fig. $2 \mathrm{~b}$ marks the transition from propagating modes to evanescent modes. In all results shown in Section 3 we have taken sufficient number of modes so that the absolute value of the elements of the first $70 \%$ of the modes do not deviate from the required values by more than the numerical values given above

\subsubsection{Evanescent energy flux contribution}

Before going further, we investigate the characteristics of the evanescent energy flux contribution from Eq. (55). The evanescent contribution term is normalised to the 
propagating Gaussian energy flux term and defined as

$$
\zeta_{=} \frac{2 \operatorname{Im} \sum_{p \in \Omega_{\mathrm{e}}^{\mathrm{I}}} \alpha_{p} r_{p}^{*}}{\sum_{p \in \Omega_{\mathrm{p}}^{\mathrm{I}}}\left|\alpha_{p}\right|^{2}} .
$$

Figure 3a shows $\zeta$ versus waveguide width for Gaussian widths between $w=0.125 \mu \mathrm{m}$ and $1 \mu \mathrm{m}$. Though the evanescent contribution varies widely with waveguide width, its maximum magnitude always decreases as the Gaussian width increases relative to the wavelength of the incident beam. As the Gaussian width is larger than the wavelength and far from the diffraction limit, the amplitude of the evanescent terms decreases, causing their energy flux contribution to decrease as well. This is further illustrated in Fig. 3b which shows the maximum $\zeta$ for each value of the width $w$. The evanescent energy flux decreases three orders of magnitude from $w=\lambda=0.5 \mu \mathrm{m}$ to $w=2 \lambda=1.0 \mu \mathrm{m}$. Note that the evanescent contribution term can be positive or negative in sign, meaning it can contribute to the forward or backward flow.

\section{Results}

The reference configuration for this study is an air to air-silver waveguide with an incident beam of wavelength $\lambda=0.5 \mu \mathrm{m}$, width $w=1 \mu \mathrm{m}$ and position centred at $l_{\mathrm{G}}=0$. The full waveguide width is $2 l=40.99 \lambda$ and is symmetric in Region II, that is $l=l_{\mathrm{d}}=l_{\mathrm{m}}=10.2475 \mu \mathrm{m}$. The permittivity of air was taken to be $\varepsilon_{d I}=\varepsilon_{d I I}=1$, and the plasmon wavelength of silver is $\lambda_{p}=144 \mathrm{~nm}$, resulting in $\varepsilon_{\mathrm{m}}=-11.0563$ at $\lambda=0.5 \mu \mathrm{m}$. For these parameters the coupling efficiency is $\eta=49 \%$, and the propagation constant of the $\mathrm{SP}$ is $\beta_{\mathrm{SP}}=13.18 \mu \mathrm{m}^{-1}$.

The square magnitude of the magnetic field for this configuration is shown in Fig. 4a with a close-up in Fig. 4b, calculated using 700 modes in each region. The incident beam propagates in Region I in the $z$-direction until it reaches Region II at $z=0$, where some of the field is transmitted and some is reflected. The transmitted field is concentrated in the dielectric $(x>0)$ near the surface of the metal $(x<0)$, as expected for a SP. The transmitted field is predominantly the SP field as the transmitted flux in the other transmitted modes is only 3.5\%. Since the Gaussian beam peaks at $x=0$, this suggests, approximately, that the half of the incident beam which overlaps with the SP field is efficiently coupled into the SP mode while the other half is reflected, consistent with Fig. 4b. In this lossless configuration, the propagating modes of the transmitted field, including the SP mode, propagate to 
$z \rightarrow \infty$. The point of concentrated field below $x=0$, the bright spot in Fig. $4 \mathrm{~b}$ indicates a corner singularity which is a common occurrence for electric field near sharp corners [32].

Figure 4a shows an extended field along the surface $-l<x<0$ at $z=0$. This phenomenon is a secondary SP which propagates transversely to the primary SP, and which originates from the point of incidence $(x=0, z=0)$. While this phenomenon was not intrinsically built into our model like the forward-propagating SP, its appearance can be substantiated by many experimental studies which have observed this phenomenon, particularly when exciting SPs near nanoslits and nanoholes [33-36]. Without the transverse SP, our results qualitatively resemble those of Stegeman et. al. The numbers of modes needed in Region I and II to observe the transverse SP are

$$
\begin{gathered}
m_{\mathrm{I}}=\frac{4 l}{\lambda} \sqrt{\frac{\varepsilon_{\mathrm{m}} \varepsilon_{\mathrm{dI}}}{\varepsilon_{\mathrm{m}}+\varepsilon_{\mathrm{dI}}}}, \\
m_{\mathrm{II}}=\frac{2}{\lambda}\left(l_{\mathrm{m}} \sqrt{\frac{\varepsilon_{\mathrm{m}} \varepsilon_{\mathrm{dI}}}{\varepsilon_{\mathrm{m}}+\varepsilon_{\mathrm{dI}}}}+l_{\mathrm{d}} \sqrt{\frac{\varepsilon_{\mathrm{m}} \varepsilon_{\mathrm{dII}}+\varepsilon_{\mathrm{dI}} \varepsilon_{\mathrm{dII}}-\varepsilon_{\mathrm{m}}^{2}}{\varepsilon_{\mathrm{m}}+\varepsilon_{\mathrm{dI}}}}\right),
\end{gathered}
$$

respectively.

These equations are derived using the observation that for the transverse SP to be properly represented, the largest $\gamma$ and $\gamma_{\mathrm{m}}$ of the modes in Region I and II need to exceed its propagation constant, $\beta_{\mathrm{SP}}$, estimated using an infinitely wide waveguide. As an example, for our typical configuration of air to air-silver with $l_{\mathrm{d}}=l_{\mathrm{m}}=10.025, m_{\mathrm{I}}=85$ and $m_{\mathrm{II}}=187$. Due to our lossless configuration, the transverse SP propagates without decay in the negative $x$-direction and reflects off the waveguide wall $(x=-l, z=0)$, causing the Fabry-Perot resonance of the total resulting field seen in Fig. 4a.

In order to see how the transverse-SP affects the coupling of the forward-propagating $\mathrm{SP}$, that is the SP of interest, the changes in coupling efficiency $\eta$ are observed for varying waveguide widths, $l$. The results in Fig. 5 are shown for the standard symmetrical air to air-silver waveguide where the waveguide width is varied, ranging between $n \lambda<2 l<(n+1) \lambda$ where $n=40-100$. Figure 5 shows that $\eta$ is oscillatory, with similar maximum and minimum values even up to waveguide widths of 100 times the wavelength. Evidently, the transverse-SP affects the coupling into the forwardSP irrespective of the position of the waveguide walls. This suggests that the effect of the transverse-SP is strongly linked to the lossless nature of the configuration, even for very large waveguides. We return to this below. 
Next, the SP coupling efficiency is determined for an asymmetric air to air-silver waveguide, that is for $l_{\mathrm{d}} \neq l_{\mathrm{m}}$. For all the following asymmetrical results, the incident beam has $l_{\mathrm{G}}=\Delta l$ so as to be always incident on the metal-dielectric interface. The coupling efficiencies are first calculated for asymmetrical waveguides with various metal widths, $l_{m}$, and with fixed dielectric width $l_{\mathrm{d}}=10.2475 \mu \mathrm{m}$. The coupling efficiencies are shown in Fig. 6a. $\eta$ is seen to be roughly periodic over the $l_{\mathrm{m}}$ range. This suggest that the periodicity is most likely associated with the Fabry-Perotlike behaviour of the transverse-SP in a cavity of length $l_{\mathrm{m}}$. Since the propagation constant of the transverse SP is $\beta_{\mathrm{SP}}=13.18 \mu \mathrm{m}^{-1}$, the period by which the coupling $\eta$ varies with the width of the metal $l_{\mathrm{m}}$ is therefore expected to be $\Delta l_{\mathrm{m}}=\pi / \beta_{\mathrm{SP}} \approx$ $0.238 \mu \mathrm{m}$. This is indicated by the red dashed vertical lines in Fig. 6a and show excellent agreement with the observed period.

It is difficult to determine the coupling efficiency into the transverse SP as the field amplitude depends on the quality factor of the transverse cavity, and so direct comparison to the amplitude of the forward-propagating SP is difficult to determine. In order to give a quantitative indication of the transverse SP energy flux, the ratio of the maximum $\left|E_{x}\right|^{2}$ of the standing wave over the incident beam is given in Fig. 6a. There is a positive correlation between the energy in the transverse SP and the coupling efficiency of the forward SP.

The coupling efficiencies were also calculated for asymmetric waveguides with various dielectric widths $l_{\mathrm{d}}$, and with a fixed metal width $l_{\mathrm{m}}=10.2475 \mu \mathrm{m}$. The coupling efficiencies are shown in Fig. 6b. The interference due to the transverse-SP does not change in these calculations as it is independent of $l_{\mathrm{d}}$. This small-amplitude periodicity in $\eta$ can be attributed to the altered number of reflection channels available every time another propagating mode appears. This in turn alters the energy flux distribution near each diffraction anomaly, a result that was observed and similarly explained by Stegeman et al. [10]. To demonstrate this, we indicate where these diffraction anomalies occur by vertical lines in Fig. 6b. Additional propagating mode in Region I are indicated in blue dashed lines and additional modes in Region II in green solid lines. Region I diffraction anomalies occur for dielectric widths $l_{\mathrm{d}}=(2 l / \lambda)\left(n+\frac{1}{2}\right), n=0,1, \ldots$, which indicates when mode transitions between propagating ( $\beta^{\mathrm{I}}$ real) and evanescent $\left(\beta^{\mathrm{I}}\right.$ imaginary). The widths at which Region II diffraction anomalies occur are found by solving Eq. (25) for $\left(\beta^{\mathrm{II}}\right)^{2}=0$ with constant $l_{\mathrm{m}}$, and are given by

$$
l_{\mathrm{d}}=\frac{1}{\sqrt{\varepsilon_{\mathrm{dII}}} k_{0}}\left(n \pi-\tan ^{-1}\left(\sqrt{\frac{\varepsilon_{\mathrm{dII}}}{\left|\varepsilon_{\mathrm{m}}\right|}} \tanh \left(\sqrt{\left|\varepsilon_{\mathrm{m}}\right|} k_{0} l_{\mathrm{m}}\right)\right)\right), n=0,1 \ldots
$$


Figure $6 \mathrm{~b}$ shows that $\eta$ is periodic about the occurrence of the diffraction anomalies. The range at which $\eta$ varies is much larger in Fig. 6a than in Fig. 6b. The relative effect of the diffraction anomalies on the range of $\eta$ values seems to be much smaller than that of the transverse-SP, suggesting that the transverse-SP is a stronger effect.

We now consider the SP coupling efficiency for different dielectrics. To do so we are guided by the approximate expression for the SP width

$$
\frac{1}{\widetilde{\gamma}_{\mathrm{dII}}}+\frac{1}{\widetilde{\gamma}_{\mathrm{m}}}=\frac{(1+\rho)}{k_{0} \rho}\left(\frac{1-\rho}{\left|\varepsilon_{\mathrm{m}}\right|}\right)^{\frac{1}{2}},
$$

where $\rho=\varepsilon_{\mathrm{dII}} /\left|\varepsilon_{\mathrm{m}}\right|$, which follows directly from the SP dispersion relation (28). Increasing the permittivity of the dielectric in Region II, thus increasing $\rho$, tends to make the SP narrower, making it more difficult to excite. Instead we increase the permittivity of the incident medium $\varepsilon_{\mathrm{dI}}$. The SP coupling efficiency versus $\varepsilon_{\mathrm{dI}}$ is shown in Fig. 7 in which both the maximum and minimum $\eta$ over the range of guide widths $10.0025<l<10.2475 \mu \mathrm{m}$ are given.

The maximum and minimum coupling efficiencies $\eta$ decrease as $\varepsilon_{\mathrm{dI}} \rightarrow 7$ from unity. However, for $7<\varepsilon_{\mathrm{dI}}<\varepsilon_{\mathrm{m}}$, the maximum grows and peaks at $\varepsilon_{\mathrm{dI}} \approx 8.5$. This peak is likely due to two competing factors. The first is that the number propagating reflection modes grow with increasing $\varepsilon_{\mathrm{dI}}$, causing maximum coupling to drop. The second factor is that the transverse SP profile becomes narrower as $\varepsilon_{\mathrm{dI}} \rightarrow\left|\varepsilon_{\mathrm{m}}\right|$, which causes maximum coupling into the forward SP to increase. From $\varepsilon_{\mathrm{dI}}=\left|\varepsilon_{\mathrm{m}}\right|$ onwards, the transverse SP ceases to exist (see Eq. (28)), indicated by the solid black line in Fig. 7. Here the coupling decreases because of the first factor. The minimum $\eta$ seem to change in an irregular manner between $7<\varepsilon_{\mathrm{dI}}<\varepsilon_{\mathrm{m}}$, suggesting it is highly sensitive to small changes. The numerical and analytical difficulties associated with calculations when $\varepsilon_{\mathrm{dI}} \rightarrow\left|\varepsilon_{\mathrm{m}}\right|$ have been discussed by Wallén et al. [37].

Until now we took $l_{\mathrm{G}}=\Delta l$, which means that the incident Gaussian beam is centred at the metal-dielectric interface in Region II. Here we consider $l_{\mathrm{G}}$ to be a free parameter which is used to optimize the coupling efficiency $\eta$. Figure 8 a shows $\eta$ versus wavelength and incident beam width. The waveguide is symmetric $\left(l=l_{d}=l_{m}\right)$, and the dielectrics in both regions are chosen to be air $\left(\varepsilon_{\mathrm{dI}}=\varepsilon_{\mathrm{dII}}=1\right)$. The wavelengthdependent silver permittivity follows from the Drude model with $\lambda_{\mathrm{p}}=144 \mathrm{~nm}$. The number of modes used is 1500 to 2000 , where more modes were used for larger waveguide widths and longer wavelengths. The $\eta$ values in Fig. 8a are the maximum coupling efficiency between waveguide widths $40 \lambda<2 l<41 \lambda$, a range which contains the maximum of a periodic cycle of $\eta$ for each wavelength-waveguide width 
pairing. The black curve in Fig. 8a gives the SP width from Eq. (61). For most of the parameters, the coupling efficiency reaches approximately $80 \%$ when the incident Gaussian beam has roughly the same width as the SP, an observation earlier made by Stegeman et al. [10] At short wavelengths the SP becomes narrower than the wavelength, which causes the matching incident beam to have substantial evanescent field components. As a consequence their contribution to the energy flux (see Eq.(57)) becomes substantial too. This is illustrated in Fig. 8b, which shows the parameter $\zeta$ defined in (57) on the same axes. For $w / \lambda>1.25$ this contribution is negligible and is not shown. For some configurations with narrow beams the contribution of the evanescent modes to the energy flux can be substantial, leading to coupling efficiencies approaching $90 \%$. We note though that this contribution rapidly varies with $\lambda$ and $w$, suggesting that the coupling efficiency is sensitive to small changes in the parameters.

Figure 8c shows the optimal beam positions for each pair of wavelengths and incident beam widths which maximise the coupling efficiencies shown in Fig. 8a. The beam positions are given as the number of wavelengths shifted towards the Region II dielectric, $l_{\mathrm{G}} / \lambda$. The results can be understood as follows: when the SP is much wider than the Gaussian or vice versa, then approximating the narrower function by a $\delta$-function it is easily seen that the overlap is largest when the narrow function coincides with the peak of the other, i.e., $l_{\mathrm{G}} \ll w, \lambda$. Moreover, at short wavelengths the SP is very narrow, also limiting the shift. The shift is therefore only substantial when both the SP and the Gaussian beam are wide, namely in the top-right corner of the figure. The shifts are all positive, which means that the beam needs to be shifted towards the dielectric because the decay rate in the dielectric is much lower than in the metal [10].

\section{Conclusions}

In this paper, we have numerically studied the coupling efficiencies of a Gaussian incident beam into a SP mode in a lossless waveguide configuration which was originally proposed by Stegeman et al. [10]. Our results differ from their results, bringing to light more detail and a previously unseen and hitherto unremarked upon phenomenon: the geometry has two metal-dielectric surfaces, on each of which a SP is excited. It is our aim to study the forward-propagating SP, around which our model is designed; in contrast, the transverse SP's excitation was unintentional. The effect of the transverse SP is inescapable. The evidence presented suggests that 
the transverse SP affects the coupling efficiency of the forward SP, leading to the large variation in SP coupling with waveguide width as the transverse SP periodically shifts in and out of resonance. Smaller variation in coupling efficiency with waveguide width are associated with anomalies when a mode transitions between propagating and evanescent [10].

In the presence of loss the transverse SP would decay during propagation weakening the Fabry-Perot resonances, thus altering the interaction with the longitudinal SP. Introducing loss into the configuration would thus allow us to determine the physical significance of the transverse-SP on the coupling efficiency, a task for future work.

Compared to numerical methods such as the finite-element method or finite-difference time-domain method with perfectly matched layers which have been used in other theory studies [17,37], our method has two advantages: (i) it has a truly infinite domain in the z-direction, and (ii) it is semi-analytical, allowing us insight into the nature of the mechanism. With the addition of loss, we would expect the effect of the artificial waveguide walls to be reduced.

While the maximum coupling efficiency numerically observed in this paper was $90 \%$, a similar figure quoted by Stegeman et al. [10], the averaged maximum coupling efficiency over the range of wavelengths tested $(0.5<\lambda<1.8 \mu \mathrm{m})$ was approximately $80 \%$. This value was also found in the theoretical study of Sun et al. using a similar geometry in which SPs between a single and a double metal-dielectric interface were coupled [4]. While the effect of removing the transverse SP is unknown until a study is carried out on a lossy configuration, we consider it likely that the theoretical maximum SP coupling efficiency from the end-fire coupling mechanism will be closer to our estimate of $80 \%$ rather than the original Stegeman et al prediction of $90 \%$.

Another difference between our study and the work of Stegeman et al is the inclusion of the contribution of evanescent modes to the energy flux. Our results suggest that this contribution can be significant as the incident beam width approaches the wavelength. This contribution term would be relevant in a physical system if the incident source was positioned close to the metal-dielectric interface, and also if the incident beam width approaches the diffraction limit. The evanescent energy contribution would also be enhanced for incident fields with discontinuities in the fields or its derivatives as these have more significant high spatial frequency components. This effect would be important in nanoscale plasmonic coupling devices. 


\section{Appendix A: Completeness Relations}

Here we derive the completeness relations

$$
\begin{gathered}
\boldsymbol{J}^{T} \boldsymbol{K}=\boldsymbol{I}, \\
\boldsymbol{J} \boldsymbol{K}^{T}=\boldsymbol{I},
\end{gathered}
$$

which we use to determine if the truncated basis of Region I modes can be expanded in the truncated basis of Region II with acceptable numerical accuracy, and vice versa. Central to the derivation are the relations

$$
\mathcal{H}_{q}^{\mathrm{II}}=\sum_{p} J_{p q} \mathcal{H}_{p}^{\mathrm{I}}
$$

and

$$
\mathcal{E}_{q}^{\mathrm{II}}=\sum_{p} K_{p q} \mathcal{E}_{p}^{\mathrm{I}}
$$

the first of which we will now derive.

We express $\mathcal{H}_{q}^{\mathrm{II}}$ in a series of the $\mathcal{H}_{p}^{\mathrm{I}}$ modes,

$$
\mathcal{H}_{q}^{\mathrm{II}}=\sum_{p} \phi_{p} \mathcal{H}_{p}^{\mathrm{I}}
$$

Multiplying Eq. (A5) by $\mathcal{E}_{p^{\prime}}^{\mathrm{I}}$ and integrating from $-l<x<l$ gives

$$
\int_{-l}^{l} \mathcal{E}_{p^{\prime}}^{\mathrm{I}} \mathcal{H}_{q}^{\mathrm{II}} d x=\sum_{p} \phi_{p} \int_{-l}^{l} \mathcal{E}_{p^{\prime}}^{\mathrm{I}} \mathcal{H}_{p}^{\mathrm{I}} d x .
$$

Using the orthogonality of the Region I modes, we see that Eq. (A6) simplifies to

$$
\phi_{p}=\int_{-l}^{l} \mathcal{E}_{p}^{\mathrm{I}} \mathcal{H}_{q}^{\mathrm{II}} d x \equiv J_{p q} .
$$

To derive the first of the completeness relations, we consider the orthogonality relation

$$
\int_{-l}^{l} \mathcal{E}_{q}^{\mathrm{II}} \mathcal{H}_{q^{\prime}}^{\mathrm{II}} d x=\delta_{q q^{\prime}}
$$


into which we substitute the representations (A3) and (A4), and exploit the Region I orthogonality relation

$$
\int_{-l}^{l} \mathcal{E}_{p}^{\mathrm{I}} \mathcal{H}_{p^{\prime}}^{\mathrm{I}} d x=\delta_{p p^{\prime}}
$$

That gives

$$
\begin{array}{r}
\int_{-l}^{l}\left(\sum_{p} K_{p q} \mathcal{E}_{p}^{I}\right)\left(\sum_{p^{\prime}} J_{p^{\prime} q^{\prime}} \mathcal{H}_{p^{\prime}}^{I}\right) d x=\delta_{q q^{\prime}}, \\
\sum_{p} K_{p q} \sum_{p^{\prime}} J_{p^{\prime} q^{\prime}} \int_{-l}^{l} \mathcal{E}_{p}^{I} \mathcal{H}_{p^{\prime}}^{I} d x=\delta_{q q^{\prime}} \\
\sum_{p} K_{p q} J_{p q^{\prime}}=\delta_{q q^{\prime}}
\end{array}
$$

which in matrix notation yields

$$
\boldsymbol{K}^{T} \boldsymbol{J}=\boldsymbol{J}^{T} \boldsymbol{K}=\boldsymbol{I}
$$

the first completeness relation (A1).

For the second of the completeness relations, we need a representation of $\mathcal{H}_{p}^{\mathrm{I}}$ and $\mathcal{E}_{p}^{\mathrm{I}}$ in terms of $\mathcal{H}_{q}^{\mathrm{II}}$ and $\mathcal{E}_{q}^{\mathrm{II}}$ bases. These are

$$
\begin{aligned}
\mathcal{H}_{p}^{\mathrm{I}} & =\sum_{q} K_{p q} \mathcal{H}_{q}^{\mathrm{II}}, \\
\mathcal{E}_{p}^{\mathrm{I}} & =\sum_{q} J_{p q} \mathcal{E}_{q}^{\mathrm{II}},
\end{aligned}
$$

which are derived in a similar manner to Eqs (A3) and (A4). Finally, by considering the orthogonality relation in (A9) and substituting in (A11) and (A12), we find that

$$
\boldsymbol{J} \boldsymbol{K}^{T}=\boldsymbol{K} \boldsymbol{J}^{T}=\boldsymbol{I},
$$

the same as relation $(53)$.

Both Eqs (A1) and (A2) hold at full rank, i.e. the inner products sum over all basis terms, and are approximately satisfied when the bases are truncated with an adequate number of modes. The quality of this approximation is demonstrated in Fig. 2. 
These relations allow us to show that, at full rank,

$$
A B=B A=I
$$

To see this, we consider

$$
\begin{aligned}
\boldsymbol{A} \boldsymbol{B} & =\left(\chi^{I}\right)^{\frac{1}{2}} \boldsymbol{J}\left(\chi^{\mathrm{II}}\right)^{-\frac{1}{2}}\left(\chi^{\mathrm{II}}\right)^{\frac{1}{2}} \boldsymbol{K}^{T}\left(\chi^{\mathrm{I}}\right)^{-\frac{1}{2}} \\
& =\left(\chi^{I}\right)^{\frac{1}{2}} \boldsymbol{J} \boldsymbol{K}^{T}\left(\chi^{\mathrm{I}}\right)^{-\frac{1}{2}} \\
& =\left(\chi^{I}\right)^{\frac{1}{2}} \boldsymbol{I}\left(\chi^{\mathrm{I}}\right)^{-\frac{1}{2}} \\
& =\boldsymbol{I}
\end{aligned}
$$

Similarly $\boldsymbol{B} \boldsymbol{A}=\boldsymbol{I}$, and so $\boldsymbol{A}=\boldsymbol{B}^{-1}$, which, at full rank, serves to reconcile the two expressions of the reflection coefficients,

$$
\begin{aligned}
& \boldsymbol{r}_{A}=\left(\boldsymbol{I}+\boldsymbol{A} \boldsymbol{A}^{T}\right)^{-1}\left(\boldsymbol{A} \boldsymbol{A}^{T}-\boldsymbol{I}\right) \boldsymbol{\alpha}, \\
& \boldsymbol{r}_{B}=\left(\boldsymbol{I}+\boldsymbol{B}^{T} \boldsymbol{B}\right)^{-1}\left(\boldsymbol{I}-\boldsymbol{B}^{T} \boldsymbol{B}\right) \boldsymbol{\alpha},
\end{aligned}
$$

and also serves to reconcile the corresponding expressions for the transmission coefficients,

$$
\begin{aligned}
& \boldsymbol{t}_{A}=2 \boldsymbol{A}^{T}\left(\boldsymbol{I}+\boldsymbol{A} \boldsymbol{A}^{T}\right)^{-1} \boldsymbol{\alpha}, \\
& \boldsymbol{t}_{B}=2 \boldsymbol{B}\left(\boldsymbol{I}+\boldsymbol{B}^{T} \boldsymbol{B}\right)^{-1} \boldsymbol{\alpha},
\end{aligned}
$$

by routine matrix manipulation.

\section{Appendix B: Energy contributions}

\subsection{Region I energy contributions}

The expression for the energy flux in Region I is

$$
S_{z}^{\mathrm{I}}=\frac{2}{Z_{0}} \operatorname{Re}\left\{\int_{-l}^{l} E_{x}^{\mathrm{I}} \hat{H}_{y}^{\mathrm{I} *} d x\right\},
$$

in which

$$
E_{x}^{\mathrm{I}}=\sum_{p}\left(\chi_{p}^{\mathrm{I}}\right)^{\frac{1}{2}} \mathcal{E}_{p}^{\mathrm{I}}\left[\alpha_{p} \exp \left(i \beta_{p}^{\mathrm{I}} z\right)-r_{p} \exp \left(-i \beta_{p}^{\mathrm{I}} z\right)\right]
$$


and

$$
\hat{H}_{y}^{\mathrm{I}}=\sum_{p}\left(\chi_{p}^{\mathrm{I}}\right)^{-\frac{1}{2}} \mathcal{H}_{p}^{\mathrm{I}}\left[\alpha_{p} \exp \left(i \beta_{p}^{\mathrm{I}} z\right)+r_{p} \exp \left(-i \beta_{p}^{\mathrm{I}} z\right)\right]
$$

Substituting Eqs (B2) and (B3) into Eq. (B1) yeilds

$$
\begin{array}{r}
S_{z}^{I}=\frac{2}{Z_{0}} \operatorname{Re}\left\{\sum_{p} \frac{\left(\beta_{p}^{\mathrm{I}}\right)^{\frac{1}{2}}}{\left(\beta_{p}^{\mathrm{I} *}\right)^{\frac{1}{2}}}\left[\alpha_{p} \exp \left(i \beta_{p}^{\mathrm{I}} z\right)-r_{p} \exp \left(-i \beta_{p}^{\mathrm{I}} z\right)\right]\right. \\
\left.\left[\alpha_{p}^{*} \exp \left(-i \beta_{p}^{\mathrm{I} *} z\right)+r_{p}^{*} \exp \left(i \beta_{p}^{\mathrm{I} *} z\right)\right]\right\},
\end{array}
$$

after exploiting the orthogonality of the modes in Region I, and realising that $\mathcal{H}_{p}^{\mathrm{I} *}=$ $\mathcal{H}_{p}^{\mathrm{I}}$ for $\beta_{p}^{\mathrm{I}}$ either purely real or imaginary. After expanding and simplifying, Eq. (B4) becomes

$$
\begin{array}{r}
S_{z}^{I}=\frac{2}{Z_{0}} \operatorname{Re}\left\{\sum_{p} \frac{\left(\beta_{p}^{\mathrm{I}}\right)^{\frac{1}{2}}}{\left(\beta_{p}^{\mathrm{I} *}\right)^{\frac{1}{2}}}\left[\left|\alpha_{p}\right|^{2} \exp \left(-2 \operatorname{Im} \beta_{p}^{\mathrm{I}} z\right)-\left|r_{p}\right|^{2} \exp \left(2 \operatorname{Im} \beta_{p}^{\mathrm{I}} z\right)\right]\right. \\
\left.+2 i \operatorname{Im}\left[\alpha_{p} r_{p} \exp \left(2 i \operatorname{Re} \beta_{p}^{\mathrm{I}} z\right)\right]\right\} .
\end{array}
$$

This sum splits into two parts depending on whether $\beta_{p}^{\mathrm{I}}$ is real or imaginary. By considering

$$
\frac{\left(\beta_{p}^{\mathrm{I}}\right)^{\frac{1}{2}}}{\left(\beta_{p}^{\mathrm{I} *}\right)^{\frac{1}{2}}}= \begin{cases}1, & \text { if } \beta_{p}^{\mathrm{I}} \text { is real, } \\ i, & \text { if } \beta_{p}^{\mathrm{I}} \text { is imaginary }\end{cases}
$$

then Eq. (B5) simplifies to the final expression for the energy flux in Region I,

$$
S_{z}^{I}=\frac{2}{Z_{0}}\left(\sum_{p=\Omega_{p}^{\mathrm{I}}}\left(\left|a_{p}\right|^{2}-\left|r_{p}\right|^{2}\right)-\sum_{p=\Omega_{e}^{\mathrm{I}}} 2 \operatorname{Im}\left\{\alpha_{p} r_{p}^{*}\right\}\right)
$$

where $\Omega_{p}^{\mathrm{I}}$ represents the set of propagating modes and $\Omega_{e}^{\mathrm{I}}$ represents the set of evanescent modes in Region I. 


\subsection{Region II energy contributions}

The expression for the energy flux from Region II is

$$
S^{\mathrm{II}}=\frac{2}{Z_{0}} \operatorname{Re}\left\{\int_{-l}^{l} E_{x}^{\mathrm{II}} \hat{H}_{y}^{\mathrm{II} *} d x\right\},
$$

given that

$$
E_{x}^{\mathrm{II}}=\sum_{q}\left(\chi_{p}^{\mathrm{II}}\right)^{\frac{1}{2}} \mathcal{E}_{p}^{\mathrm{II}} t_{q} \exp \left(i \beta_{p}^{\mathrm{II}} z\right)
$$

and

$$
\hat{H}_{q}^{\mathrm{II}}=\sum_{q}\left(\chi_{q}^{\mathrm{II}}\right)^{-\frac{1}{2}} \mathcal{H}_{q}^{\mathrm{II}} t_{q} \exp \left(i \beta_{q}^{\mathrm{II}} z\right)
$$

Substituting Eqs (B9) and (B10) into Eq. (B8) gives

$$
S_{z}^{\mathrm{II}}=\frac{2}{Z_{0}} \operatorname{Re}\left\{\sum_{q} \sum_{q^{\prime}} \frac{\left(\beta_{q}^{\mathrm{II}}\right)^{\frac{1}{2}}}{\left(\beta_{q^{\prime}}^{\mathrm{II} *}\right)^{\frac{1}{2}}} t_{q} t_{q^{\prime}}^{*} \exp \left[i\left(\beta_{q}^{\mathrm{II}}-\beta_{q^{\prime}}^{\mathrm{II} *}\right) z\right] \int_{-l}^{l} \mathcal{E}_{q}^{\mathrm{II}} \mathcal{H}_{q^{\prime}}^{\mathrm{II} *} d x\right\} .
$$

In order to deal with the more complicated $\mathcal{E}^{\mathrm{II}}$ and $\mathcal{H}^{\mathrm{II}}$ forms of Region II compared to the equivalent expressions of Region I, we shall express the orthogonality condition of Region II as

$$
\int_{-l}^{l} \mathcal{E}_{\beta_{q}}^{\mathrm{II}} \mathcal{H}_{\beta_{q^{\prime}}}^{\mathrm{II}} d x=\delta_{\beta_{q} \beta_{q^{\prime}}}
$$

in which $\mathcal{E}_{\beta_{q}}^{\mathrm{II}}$ refers to $\mathcal{E}_{q}^{\mathrm{II}}$ where $q$ indexes the mode for which $\beta^{\mathrm{II}}=\beta_{q}^{\mathrm{II}}$, and similarly for $\mathcal{H}_{\beta_{q^{\prime}}}^{\mathrm{II}}$.

Going further, we note that the only complex entities in the expressions for $\mathcal{E}_{\beta_{q}}^{\mathrm{II}}$ and $\mathcal{H}_{\beta_{q}}^{\mathrm{II}}$ are $\widetilde{\gamma}_{\mathrm{dII}}$ and $\widetilde{\gamma}_{\mathrm{m}}$. The relation between taking the complex conjugate of $\widetilde{\gamma}_{\mathrm{dII}}$ and $\widetilde{\gamma}_{\mathrm{m}}$ respectively with the complex conjugate of $\beta_{p}^{\mathrm{II}}$ are

$$
\widetilde{\gamma}_{\mathrm{dII}, \beta_{q}^{\mathrm{II}}}^{*}=\left\{\left[\left(\beta_{p}^{\mathrm{II}}\right)^{2}-\varepsilon_{\mathrm{dII}} k_{0}\right]^{\frac{1}{2}}\right\}^{*}=\left[\left(\beta_{p}^{\mathrm{II} *}\right)^{2}-\varepsilon_{\mathrm{dII}} k_{0}\right]^{\frac{1}{2}}=\widetilde{\gamma}_{\mathrm{dII}, \beta_{q}^{\mathrm{II} *}},
$$

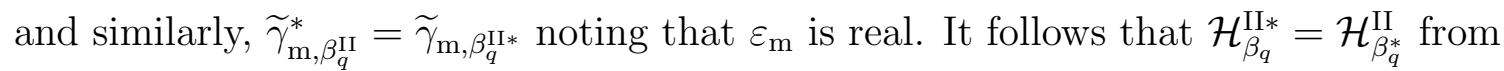
which the form of the orthogonality relation (A8) reduces to

$$
\int_{-l}^{l} \mathcal{E}_{\beta_{q}}^{\mathrm{II}} \mathcal{H}_{\beta_{q^{\prime}}^{*}}^{\mathrm{II}} d x=\delta_{\beta_{q} \beta_{q^{\prime}}^{*}}
$$


In other words, we see from Eq. (B14) that a non-zero energy contribution to Eq. (B11) requires that $\beta_{q}^{\mathrm{II}}=\beta_{q^{\prime}}^{\mathrm{II} *}$. Region II $\beta_{q}^{\mathrm{II}}$ can be either purely real, purely imaginary or occur in pairs with the same positive imaginary part and equal real parts with opposite signs i.e $\beta_{q}^{\mathrm{II}}$ and $-\beta_{q}^{\mathrm{II} *}$. The combinations of $\beta_{q}^{\mathrm{II}}$ and $\beta_{q^{\prime}}^{\mathrm{II}}$ are given in Table 2, and the possible non-zero energy contributions are marked with a $\checkmark$. Each of these combinations must be investigated separately.

\subsubsection{Real $\beta_{q}^{\mathrm{II}}$}

For real $\beta_{q}^{\mathrm{II}}$ values, i.e. for all the propagating modes of Region II, then $\beta_{q}^{\mathrm{II}}=\beta_{q}^{\mathrm{II} *}$. It follows that the contribution to $S_{z}^{\mathrm{II}}$ is

$$
S_{z}^{\mathrm{II}}=\frac{2}{Z_{0}} \operatorname{Re}\left\{\sum_{q \in \Omega_{p}^{\mathrm{II}}}\left|t_{q}\right|^{2}\right\} .
$$

\subsubsection{Imaginary $\beta_{q}^{\mathrm{II}}$}

For imaginary $\beta_{q}^{\mathrm{II}}, \beta_{q}^{\mathrm{II} *}=-\beta_{q}^{\mathrm{II}}$, we have $\widetilde{\gamma}_{\mathrm{dII}, \beta_{q}^{\mathrm{II}}}^{*}=\widetilde{\gamma}_{\mathrm{dII},\left(-\beta_{q}^{\mathrm{II} *}\right)}$, and similarly $\widetilde{\gamma}_{\mathrm{m}, \beta_{q}^{\mathrm{II}}}^{*}=$ $\widetilde{\gamma}_{\mathrm{m},\left(-\beta_{q}^{\mathrm{II} *}\right)}$, which leads to $\mathcal{H}_{q}^{\mathrm{II} *}=\mathcal{H}_{q}^{\mathrm{II}}$. Here, the contribution to $S_{z}^{\mathrm{II}}$ by the imaginary $\beta_{q}^{\mathrm{II}}$ modes is

$$
\begin{aligned}
S_{z}^{\mathrm{II}} & =\frac{2}{Z_{0}} \operatorname{Re}\left\{\sum_{q} i\left|t_{q}\right|^{2} \exp \left(-2 \operatorname{Im} \beta_{q}^{\mathrm{II}} z\right)\right\} \\
& =0 .
\end{aligned}
$$

Thus, there is no energy contribution from modes with purely imaginary $\beta_{q}^{\mathrm{II}}$.

\subsubsection{Complex $\beta_{q}^{\mathrm{II}}$}

For the case of the complex $\beta_{q}^{\mathrm{II}}$ case, we need to consider the two possible pairs of modes $\left(\beta_{q}^{\mathrm{II}},-\beta_{q}^{\mathrm{II} *}\right)$ and $\left(-\beta_{q}^{\mathrm{H} *}, \beta_{q}^{\mathrm{II}}\right)$, according to Table 2 . Again, we see that $\widetilde{\gamma}_{\mathrm{dII},\left(-\beta_{q}^{\mathrm{II} *}\right)}=\widetilde{\gamma}_{\mathrm{dII},\left(\beta_{q}^{\mathrm{II}}\right)}^{*}$ and similarly $\widetilde{\gamma}_{\mathrm{m},\left(-\beta_{q}^{\mathrm{II} *}\right)}=\widetilde{\gamma}_{\mathrm{m},\left(\beta_{q}^{\mathrm{II}}\right)}^{*}$. This causes $\mathcal{H}_{-\beta_{q}^{\mathrm{II} *}}=\mathcal{H}_{\beta_{q}^{\mathrm{II} *}}=$ 
$\mathcal{H}_{\beta_{q}^{\mathrm{II}}}^{*}$. The cross-term between $\beta_{q}^{\mathrm{II}}$ and $-\beta_{q^{\prime}}^{\mathrm{II} *}$ becomes

$$
\int_{-l}^{l} \mathcal{E}_{\beta_{q}^{\mathrm{II}}} \mathcal{H}_{-\beta_{q^{\prime}}^{\mathrm{II} *}}^{*} d x=\int_{-l}^{l} \mathcal{E}_{\beta_{q}^{\mathrm{II}}} \mathcal{H}_{\beta_{q^{\prime}}^{\mathrm{II}}} d x=\delta_{\beta_{q}^{\mathrm{II}} \beta_{q^{\prime}}^{\mathrm{II}},}
$$

and similarly for the cross-term in the integral of Eq. (B11) between $-\beta_{q}^{\text {II* }}$ and $\beta_{q^{\prime}}^{\mathrm{II}}$

So both combinations of $\beta_{q}^{\mathrm{II}}$ and $\beta_{q}^{\mathrm{II} *}$ give possible non-zero energy contributions. The contribution of the pair to $S_{z}^{\mathrm{II}}$ is then

$$
\begin{aligned}
S_{z}^{\mathrm{II}}=\frac{2}{Z_{0}} \operatorname{Re} & \left\{\frac{\left(\beta_{q}^{\mathrm{II}}\right)^{\frac{1}{2}}}{\left(-\beta_{q}^{\mathrm{II} *}\right)^{\frac{1}{2} *}} t_{\beta_{q}^{\mathrm{II}}}^{*} t_{-\beta_{q}^{\mathrm{II} *}} \exp \left[i\left(\beta_{q}^{*}-\left(-\beta_{q}^{\mathrm{II} *}\right)\right) z\right]+\right. \\
& \left.\frac{\left(-\beta_{q}^{\mathrm{II} *}\right)^{\frac{1}{2}}}{\left(\beta_{q^{\prime}}^{\mathrm{II}}\right)^{\frac{1}{2} *}} t_{-\beta_{q}^{\mathrm{II} *} t_{\beta_{q}}^{*}} \exp \left[i\left(-\beta_{q}^{\mathrm{II} *}-\left(\beta_{q}^{\mathrm{II}}\right)^{*}\right) z\right]\right\} .
\end{aligned}
$$

Since

$$
\frac{\left(\beta_{q}^{\mathrm{II}}\right)^{\frac{1}{2}}}{\left(-\beta_{q^{\prime}}^{\mathrm{II} *}\right)^{\frac{1}{2} *}}=\frac{\left(-\beta_{q}^{\mathrm{II} *}\right)^{\frac{1}{2}}}{\left(\beta_{q^{\prime}}^{\mathrm{II}}\right)^{\frac{1}{2} *}}=i
$$

it follows that the contributions to $S_{z}^{\mathrm{II}}$ for the cross-coupled pairs of modes is

$$
\begin{aligned}
S_{z}^{\mathrm{II}} & =\frac{2}{Z_{0}} \operatorname{Re}\left\{i t_{\beta_{q}^{\mathrm{II}}}^{*} t_{-\beta_{q}^{\mathrm{II} *}} \exp \left(2 i \beta_{q}^{\mathrm{II}} z\right)+i t_{-\beta_{q}^{\mathrm{II} *}} t_{\beta_{q}^{\mathrm{II}}}^{*} \exp \left(-2 i \beta_{q}^{\mathrm{II} *} z\right)\right\} \\
& =\frac{2}{Z_{0}} \operatorname{Re}\left\{2 i \operatorname{Re}\left[t_{\beta_{q}^{\mathrm{II}}} t_{-\beta_{q}^{\mathrm{II} *}} \exp \left(2 i \beta_{q}^{\mathrm{II}} z\right)\right]\right\} \\
& =0
\end{aligned}
$$

Thus, there is no energy contribution from modes with complex $\beta_{q}^{\mathrm{II}}$.

Accordingly, the total energy flux in Region II is

$$
S_{z}^{\mathrm{II}}=\frac{2}{Z_{0}} \sum_{q=\Omega_{p}^{\mathrm{II}}}\left|t_{q}\right|^{2},
$$

where $\Omega_{p}^{\mathrm{II}}$ is the set of propagating modes of Region II. The conservation of energy flux result in Eq. (54) in the main paper follows from Eqs (B7) and (B20). 


\section{Acknowledgements}

This research was supported by the Australian Research Council (ARC) Centre of Excellence for Ultrahigh Bandwidth Devices for Optical Systems (CE110001018).

\section{References}

1. P. Berini, "Bulk and surface sensitivities of surface plasmon waveguides," New Journal of Physics 10(10), 105010 (2008).

2. P. Berini, "Long-range surface plasmon polaritons," Advances in Optics and Photonics 1(3), 484-588 (2009).

3. G. I. Stegeman and C. T. Seaton, "Nonlinear surface plasmons guided by thin metal films," Optics Letters 9(6), 235-7 (1984).

4. Z. Sun and D. Zeng, "Coupling of surface plasmon waves in metal/dielectric gap waveguides and single interface waveguides," Journal of the Optical Society of America B 24(11), 2883-7 (2007).

5. H. T. M. C. M. Baltar, K. Drozdowicz-Tomsia, and E. M. Goldys, "Propagating surface plasmons and dispersion relations for nanoscale multiplayer metallicdielectric films," in Plasmonics-Principles and Applications , K. Y. Kim, ed. (InTech, 2012), Chap. 6, pp. 136-55.

6. A. Otto, "Excitation of nonradiative surface plasmon waves in silver by method of frustrated total reflection," Zeitschrift für Physik 216(4), 398-410 (1968).

7. E. Kretschmann, "Die bestimmungen optischer Konstanten von Metallen durch Anregung van Oberflächen plasma-schwingungen," Zeitschrift für Physik 241(4), 313-24 (1971).

8. G. S. Agarwal, "Electromagnetic scattering, local field enhancements, and longrange surface plasmons in layered structures with roughness," Physical Review B 31(6), 3534-39 (1985).

9. L. Wendler and R. Haupt, "An improved virtual mode theory of ATR experiments on surface polaritons," Physica Status Solidi (b) 143(1), 131-48 (1987).

10. G. I. Stegeman, R. F. Wallis, and A. A. Maradudin, "Excitation of surface polaritons by end-fire coupling." Optics Letters 8(7), 386-8 (1983). 
11. R. Charbonneau, P. Berini, E. Berolo, and E. Lisicka-Shrzek, "Experimental observation of plasmon-polariton waves supported by a thin metal film of finite width," Optics Letters 25(11), 844-6 (2000).

12. Y. Kurokawa and H. Miyazaki, "Metal-insulator-metal plasmon nanocavities: analysis of optical properties," Physical Review B 75(3), 035411 (2007).

13. Y. Song, J. Wang, Q. Li, M. Yan, and M. Qiu, "Broadband coupler between silicon waveguide and hybrid plasmonic waveguide." Optics Express 18(12), 13173 9 (2010).

14. C. S. Kim, I. Vurgaftman, R. A. Flynn, M. Kim, J. R. Lindle, W. W. Bewley, K. Bussmann, J. R. Meyer, and J. P. Long, "An integrated surface-plasmon source." Optics Express 18(10), 10609-15 (2010).

15. J. Gosciniak, T. Holmgaard, and S. I. Bozhevolnyi, "Theoretical analysis of long-range dielectric-loaded surface plasmon polariton waveguides," Journal of Lightwave Technology 29(10), 1473-81 (2011).

16. S.-Y. Lee, J. Park, M. Kang, and B. Lee, "Highly efficient plasmonic interconnector based on the asymmetric junction between metal-dielectric-metal and dielectric slab waveguides." Optics Express 19(10), 9562-74 (2011).

17. X.-W. Chen, V. Sandoghdar, and M. Agio, "Nanofocusing radially-polarized beams for high-throughput funneling of optical energy to the near field." Optics Express 18(10), 10,878-87 (2010).

18. H.-J. Lim, M.-S Kwon, "Efficient coupling between photonic and dielectricloaded surface plasmon polariton waveguides with the same core material," IEEE Photonics Journal 6(3), 1-9 (2014).

19. J. Jahns, L. Moeller, A. Edelmann, "Coupling of terahertz radiation to metallic wire using end-fire technique," Electronic Letters, 49(14) 884-6 (2013).

20. X.-W. Chen, V. Sandoghdar, and M. Agio, "Highly efficient interfacing of guided plasmons and photons in nanowires." Nano Letters 9(11), 3756-61 (2009).

21. M. A. Schmidt, L. N. Prill Sempere, H. K. Tyagi, C. G. Poulton, and P. S. J. Russell, "Waveguiding and plasmon resonances in two-dimensional photonic lattices of gold and silver nanowires," Physical Review B 77(3), 033417 (2008).

22. S. J. Al-Bader, "Optical transmission on metallic wires-fundamental modes," IEEE Journal of Quantum Electronics 40(3), 325-29 (2004). 
23. R. Charbonneau, N. Lahoud, G. Mattiussi, and P. Berini, "Demonstration of integrated optics elements based on long-ranging surface plasmon polaritons," Optics Express 13(3), 977-84 (2005).

24. R. Charbonneau, C. Scales, I. Breukelaar, S. Fafard, N. Lahoud, G. Mattiussi, and P. Berini, "Passive integrated optics elements based on long-range surface plasmon polaritons," Journal of Lightwave Technology 24(1), 477-94 (2006).

25. H. Hu, X. Zeng, D. Ji, L. Zhu, Q. Gan, "Efficient end-fire coupling of surface plasmons on flat metal surfaces for improved plasmonic Mach-Zehnder interferometer," Journal of Applied Physics, 113(5), 053101 (2013).

26. A. A. Maradudin, R. F. Wallis, and G. I. Stegeman, "The optics of surface and guided wave polaritons," Progress in Surface Science 33(3), 171-257 (1990).

27. B. Sturman, E. Podivilov, and M. Gorkunov, "Eigenmodes for metal-dielectric light-transmitting nanostructures," Physical Review B 76, 125104 (2007).

28. B. Sturman, E. Podivilov, and M. Gorkunov, "Eigenmodes for the problem of extraordinary light transmission through subwavelength holes," Europhysics Letters 80(12), 24002 (2007).

29. P. Berini, "Plasmon-polariton waves guided by thin lossy metal films of finite width: Bound modes of symmetric structures," Physical Review B 61(15), 10484$503(2000)$.

30. L. C. Botten and R. C. McPhedran, "Completeness and modal expansion methods in diffraction theory," Optica Acta 32(12), 1479-88 (1985).

31. K. B. Dossou, L. C. Botten, A. A. Asatryan, B. C. P. Sturmberg, M. A. Byrne, C. G. Poulton, R. C. McPhedran, and C. M. de Sterke, "Modal formulation for diffraction by absorbing photonic crystal slabs," Journal of the Optical Society of America A 29(5), 817-31 (2012).

32. J. Meixner, "The behavior of electromagnetic fields at edges," IEEE Transactions on Antennas and Propagation 20(4), 442-46 (1972).

33. G. Gay, O. Alloschery, B. Viaris de Lesegno, C. ODwyer, J. Weiner, and H. J. Lezec, "The optical response of nanostructured surfaces and the composite diffracted evanescent wave model," Nature Physics 2(4), 262-67 (2006).

34. K. M. Goodfellow, R. Beams, C. Chitraleema, L. Novotny, and A. N. Vamivakas, "Integrated nanophotonics based on nanowire plasmons and atomically thin material," Optica 1(3), 149-52 (2014). 
35. R. Gordon, D. Sinton, K. L. Kavanagh, and A. G. Brolo, "Extraordinary optical transmission," Accounts of Chemical Research 41(8), 1049-57 (2008).

36. J. Li, C. Yang, J. Li, Z. Li, S. Zu, S. Song, H. Zhao, F. Lin, and X. Zhu, "Plasmonic focusing in nanostructures," Plasmonics 9(4), 879-86 (2014).

37. H. Wallén, H. Kettunen, and A. Sihvola, "Surface modes of negative-parameter interfaces and the importance of rounding sharp corners," Metamaterials 2, 11321 (2008). 
Table 1: Characteristics of the four types of modes in Region II

\begin{tabular}{|c|c|c|c|c|}
\hline Case & $\left(\beta^{\mathrm{II}}\right)^{2}$ & $\beta^{\mathrm{II}}$ & $\widetilde{\gamma}_{\mathrm{dII}}$ & $\widetilde{\gamma}_{\mathrm{m}}$ \\
\hline \hline I & $\begin{array}{c}\text { positive real } \\
\left(\beta^{\mathrm{II}}\right)^{2}>\varepsilon_{\mathrm{dII}} k_{0}^{2}\end{array}$ & $\begin{array}{c}\text { real } \\
\beta^{\mathrm{II}}>\sqrt{\varepsilon_{\mathrm{dII}}} k_{0}\end{array}$ & real & real \\
\hline II & positive real & real & imaginary & real \\
& $0<\left(\beta^{\mathrm{II}}\right)^{2}<\varepsilon_{\mathrm{dII}} k_{0}^{2}$ & $0<\beta^{\mathrm{II}}<\sqrt{\varepsilon_{\mathrm{dII}}} k_{0}$ & & \\
\hline III & negative real & imaginary & imaginary & real \\
& $\varepsilon_{\mathrm{m}} k_{0}^{2}<\left(\beta^{\mathrm{II}}\right)^{2}<0$ & $\mathrm{i} \sqrt{\left|\varepsilon_{\mathrm{m}}\right|} k_{0}<\beta^{\mathrm{II}}<0$ & & \\
\hline IV & negative real, & imaginary, & imaginary, & imaginary, \\
& complex pairs & complex pairs & complex & complex \\
& $\left(\beta_{\mathrm{II}}\right)^{2}<\varepsilon_{\mathrm{m}} k_{0}^{2}$ & $\mathrm{i} \sqrt{\left|\varepsilon_{\mathrm{m}}\right|} k_{0}<\beta_{\mathrm{II}}$ & & \\
\hline
\end{tabular}


Table 2: Possible energy flux contribution terms of Region II

\begin{tabular}{|c|c|c|c|c|}
\hline & real $\beta_{q}^{\text {II }}$ & imaginary $\beta_{q}^{\text {II }}$ & complex $\beta_{q}^{\text {II }}$ & complex $-\beta_{q}^{\text {II* }}$ \\
\hline real $\beta_{q^{\prime}}^{\text {II }}$ & $\checkmark$ & 0 & 0 & 0 \\
\hline imaginary $\beta_{q^{\prime}}^{\text {II }}$ & 0 & $\checkmark$ & 0 & 0 \\
\hline complex $\beta_{q^{\prime}}^{I I}$ & 0 & 0 & 0 & $\checkmark$ \\
\hline complex $-\beta_{q^{\prime}}^{\text {II }}$ & 0 & 0 & $\checkmark$ & 0 \\
\hline
\end{tabular}




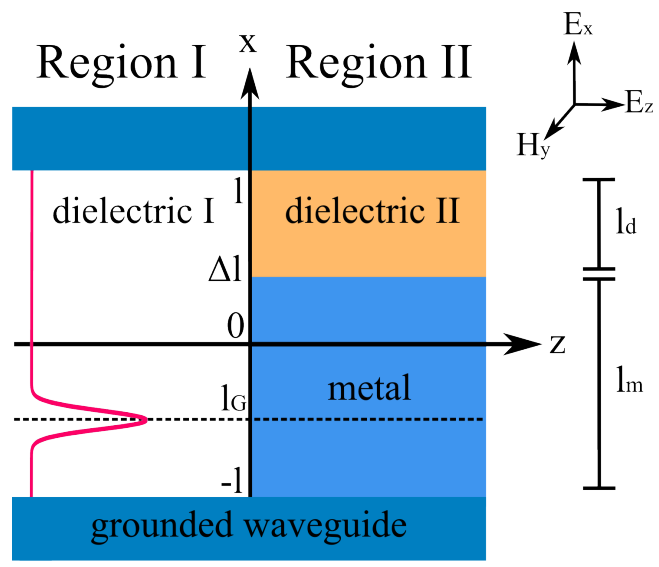

Figure 1: End-fire coupling configuration in a metal waveguide. The red curve represents the field of the incident Gaussian beam. 

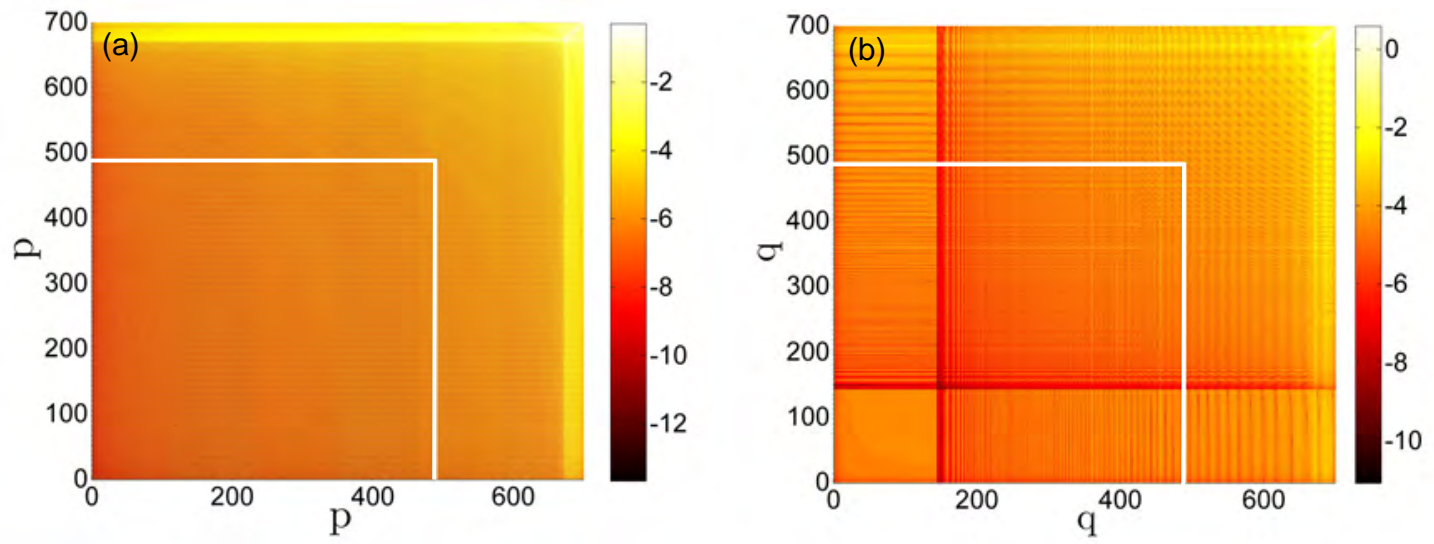

Figure 2: Logarithm base 10 of the absolute value of the elements of (a) $\boldsymbol{J}^{T} \boldsymbol{K}-\boldsymbol{I}$, and (b) $\boldsymbol{J} \boldsymbol{K}^{T}-\boldsymbol{I}$. Each figure refers to a geometry with an air to air-silver waveguide with $l=l_{\mathrm{d}}=l_{\mathrm{m}}=10.2475 \mu \mathrm{m}$, and $\lambda=0.5 \mu \mathrm{m}$ with $P=Q=700$. The solid white lines indicate the boundary of the first $70 \%$ of the modes. 

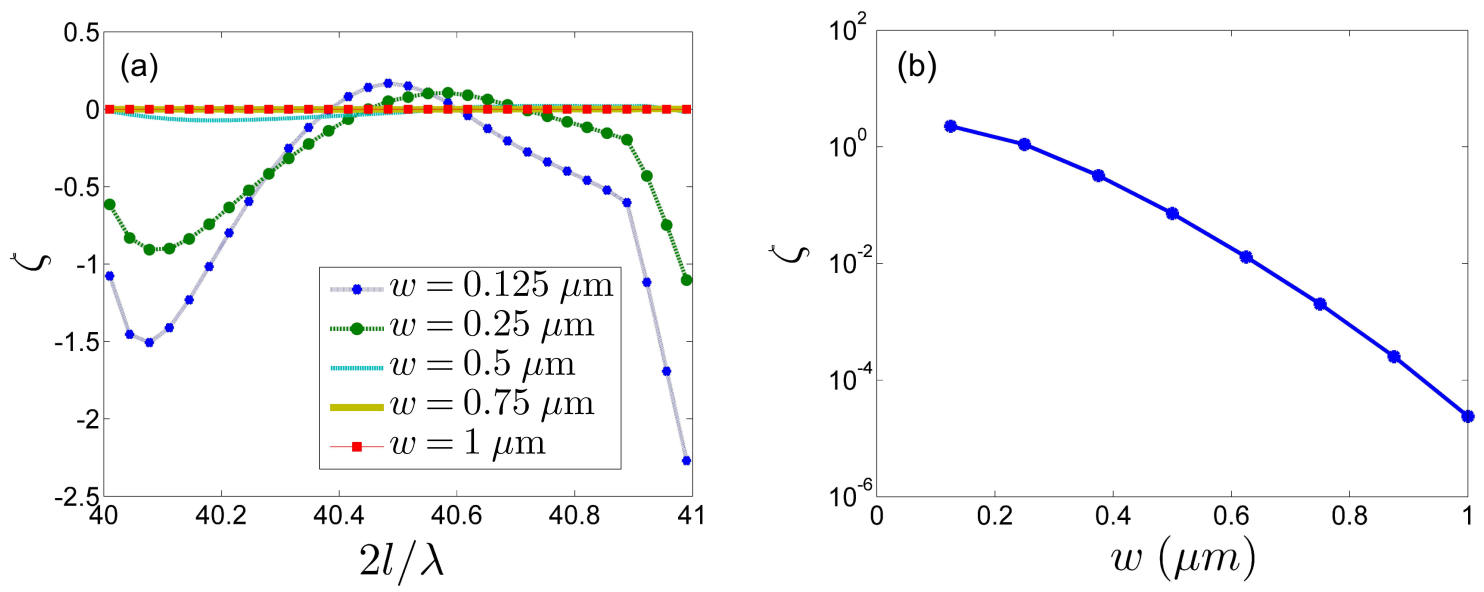

Figure 3: (a) Variation in the evanescent energy flux contribution with waveguide width, incident beam widths of $w=0.125-1 \mu \mathrm{m}$ for an air to air-silver symmetrical waveguide with $l=l_{d}=l_{m}$, and an incident beam with $\lambda=0.5 \mu \mathrm{m}$, centred at $l_{\mathrm{G}}=0$. (b) Maximum values of evanescent energy flux contribution normalised to the incident energy flux in the propagating modes. 

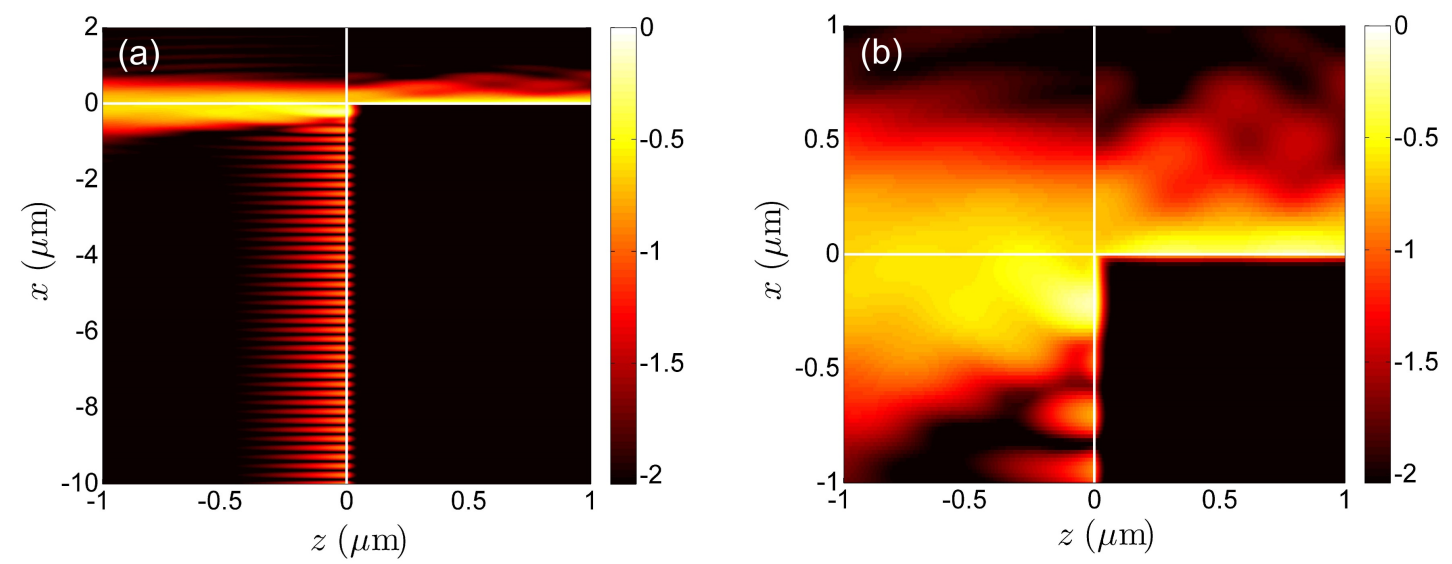

Figure 4: (a) $\log _{10}\left|H_{y}\right|^{2}$ normalised to the maximum value of $\left|H_{y}\right|^{2}$. (b) Close-up of the corner where the SP is launched. Here, $-1 \mu \mathrm{m}<z<1 \mu \mathrm{m}$ and $-l<x<2 \mu \mathrm{m}$. (b) A close-up of the corner singularity feeding the SP. In this example $\varepsilon_{\mathrm{dI}}=\varepsilon_{\mathrm{dII}}=1$ and $\varepsilon_{\mathrm{m}}=-11.0563$, corresponding to silver at $\lambda=0.5 \mu \mathrm{m}$. Further, $l=l_{d}=$ $l_{m}=10.2475 \mu \mathrm{m}$, and the incident beam is a Gaussian centred at $x=0$ and with $w=1 \mu \mathrm{m}$. 700 modes are used in each region. The white lines mark $x=0$ and $z=0$. 


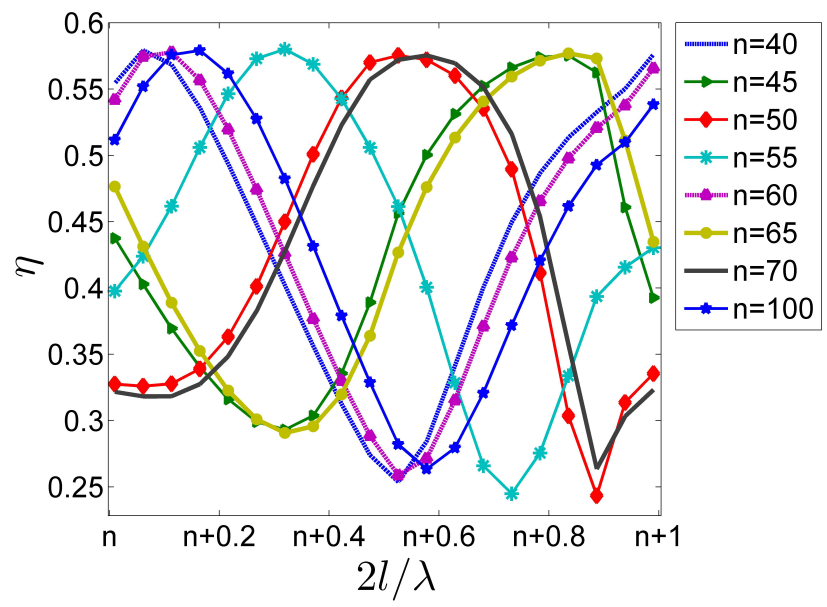

Figure 5: SP coupling efficiency for varying waveguide widths in the reference air to air-silver waveguide coupling configuration. The result is calculated with 700-1100 modes in each region, where more modes are used for larger guide widths. 

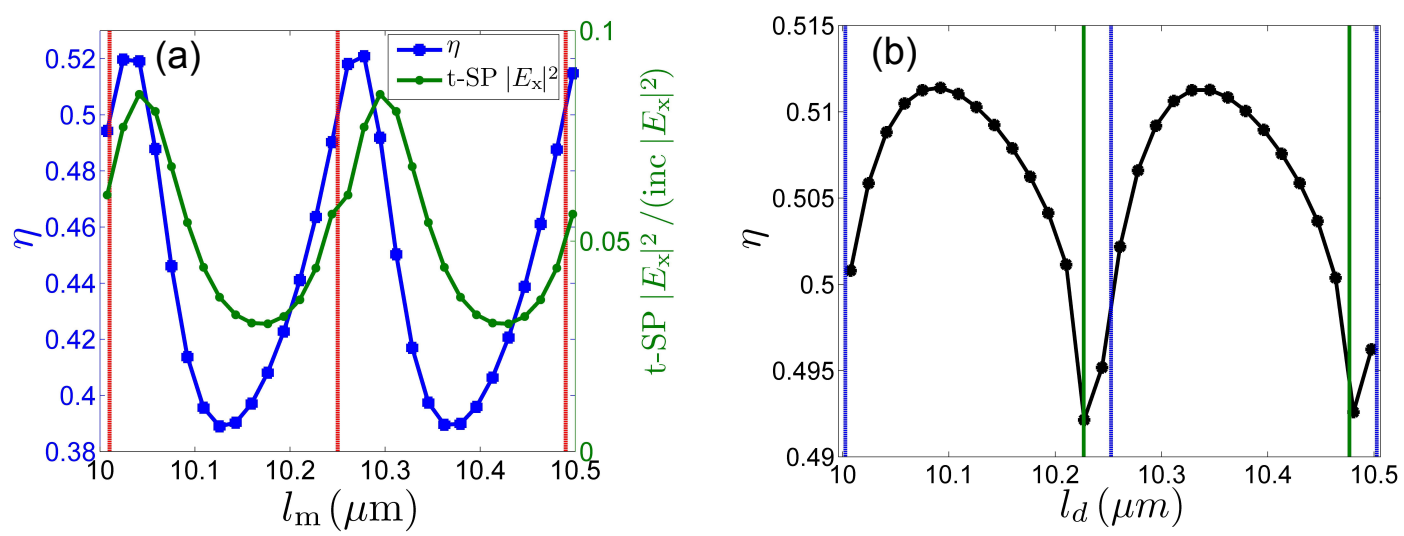

Figure 6: (a) (left vertical axis) SP coupling efficiency $\eta$ for varying metal widths $l_{\mathrm{m}}$. The fixed dielectric width is $l_{\mathrm{d}}=10.2475 \mu \mathrm{m}$. The period boundaries predicted by $\gamma$ of the transverse SP are indicated by the vertical red dashed lines. (right vertical axis) Ratio of maximum $\left|E_{x}\right|^{2}$ of the transverse SP over that of the incident field. (b) $\eta$ for varying Region II dielectric widths $l_{\mathrm{d}}$. The fixed metal width is $l_{\mathrm{m}}=10.2475 \mu \mathrm{m}$. The vertical lines indicate when an extra Region I (blue dashed) or Region II (green solid) propagating mode appears. The waveguide for both (a) and (b) have total width $2 l=l_{\mathrm{d}}+l_{\mathrm{m}}$, and $l_{\mathrm{G}}=\Delta l$. 


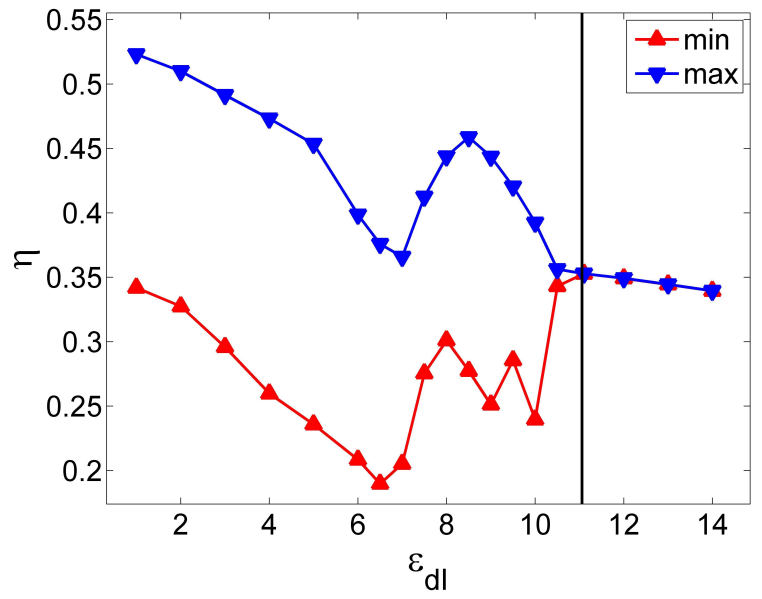

Figure 7: Maximum and minimum $\eta$ values in the range of waveguide widths $10.0025 \mu \mathrm{m}<l<10.2475 \mu \mathrm{m}$ versus $\varepsilon_{\mathrm{dI}}$. The other parameters are that of the standard configuration. 

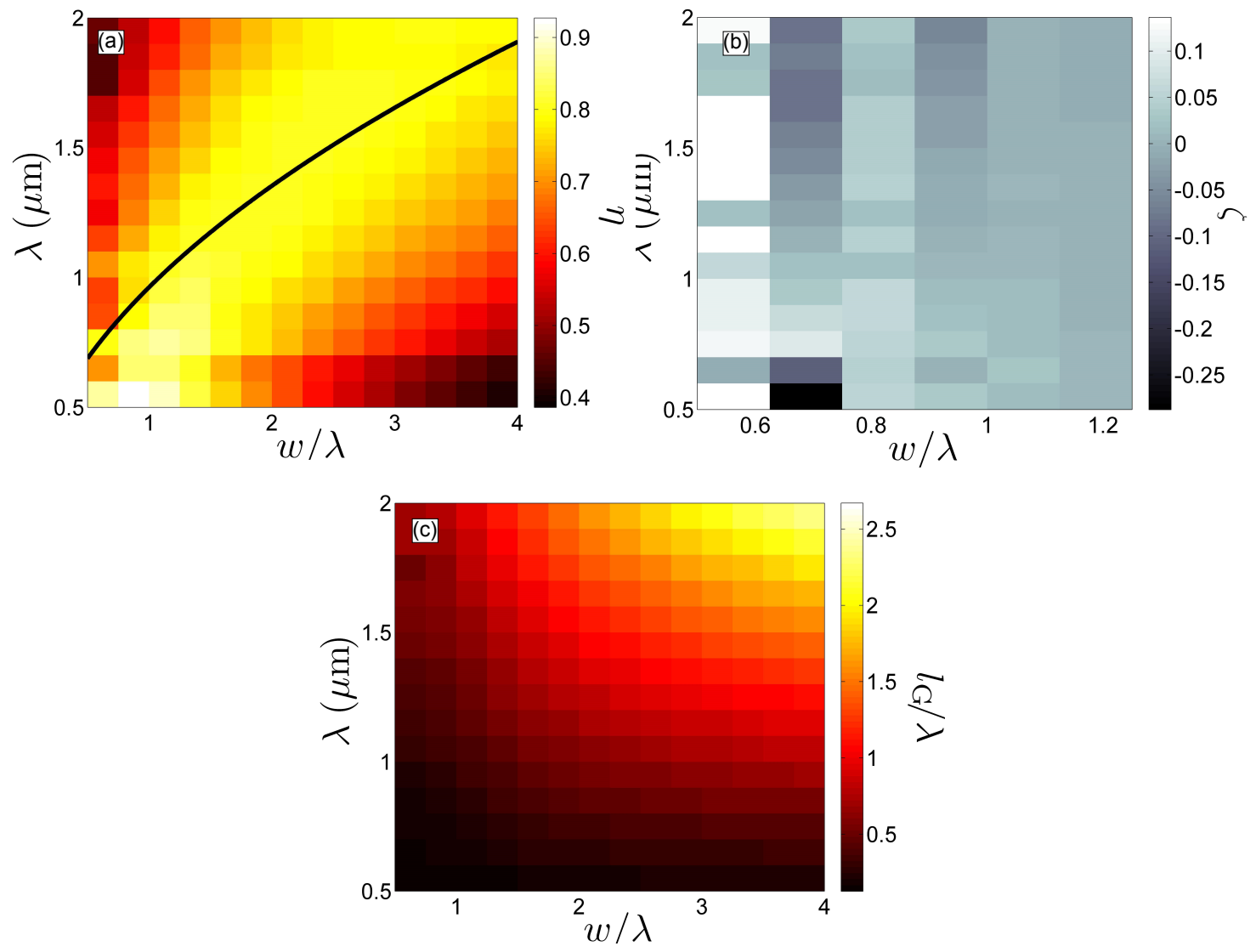

Figure 8: (a) Maximum SP coupling efficiency $\eta$ versus the normalized width of the incident Gaussian beam $w / \lambda$ and wavelength $\lambda$. In each case the coupling is optimized by suitable choice of the shift of the input beam $l_{\mathrm{G}}$ and waveguide width in the range $40 \lambda<2 l<41 \lambda$. The solid black line indicates the width of the SP following from (28). (b) Evanescent energy contribution $\zeta$ for each data point in part (a) For $w / \lambda>1.25$ this contribution is negligible and the data is not shown. (c) Shift in beam position corresponding to each SP coupling efficiency given in (a). The shifts are into the Region II dielectric. 